\title{
Lipschitz Spaces and Bernstein's Theorem on Absolutely Convergent Fourier Transforms*
}

\author{
C. S. HERZ
}

Communicated by M. KAC

0. Introduction. The two most important results involving Lipschitz spaces are Bernstein's Theorem and Sobolev's Lemma. They are statements of the following form.

Bernstein's Theorem. $\mathcal{F}: \mathbf{\Lambda} \rightarrow \mathrm{L}_{p}$ (F signifies Fourier transform);

Sobolev's Lemma. $\mathbf{\Lambda} \subset \mathbf{L}_{a}$, where $\mathbf{\Lambda}$ is an appropriate Lipschitz space.

There are various notions of "Lipschitz space" which have been considered. We shall introduce spaces $\boldsymbol{\Lambda}_{a, p}^{\alpha}$ which are the simplest to define and yield the most powerful theorems. Our spaces $\boldsymbol{\Lambda}_{a, p}^{\alpha}$ are closely related to the spaces $\Lambda(\alpha ; a, p)$ studied by Taibleson [6]. When $\alpha>0, \Lambda(\alpha ; a, p)=\mathbf{\Lambda}_{a, p}^{\alpha} \cap \mathbf{L}_{a}$; the full details will be found in Appendix 2.

The precise definition of $\boldsymbol{\Lambda}_{a, p}^{\alpha}$ is given at the beginning of Section 1. There are, up to equivalence, many ways of defining the $\boldsymbol{\Lambda}_{a, p}^{\alpha}$-norm. The easiest approach is to use a concrete definition in terms of differences. Given a function $f$, a positive integer $k$, and $1 \leqq a \leqq \infty$ we put $\omega(h)=\left\|\Delta^{k}(h) f\right\|_{a}$ where $\Delta^{k}(h)$ is the $k$ th difference operator. For $0<\alpha<k$ we define $\Lambda_{a, p}^{\alpha}(f)$ to be the $\mathscr{L}_{p}$-norm (using the dilation-invariant measure on $R^{n}-\{0\}$ in $h$ of $|h|^{-\alpha} \omega(h)$. Note that the $\mathbf{\Lambda}_{a, p}^{\alpha}$-norm depends only on (arbitrarily high order) differences of the function. The $\boldsymbol{\Lambda}_{a, p}^{\alpha}$ spaces with $\alpha>0$ are the Banach spaces obtained by completing $\mathfrak{D}$ (infinitely differentiable functions of compact support) for the $\mathbf{\Lambda}_{a, p^{-}}^{\alpha}$ norm. The basic facts about the $\boldsymbol{\Lambda}_{a, p}^{\alpha}$ spaces for $\alpha>0$ are given in $\$ 1$. It is technically convenient to extend the definition to $\alpha \leqq 0$; this is done in $\$ 6$ where we show that $f \boldsymbol{\varepsilon} \mathbf{\Lambda}_{a, p}^{\alpha}$ iff its derivatives of order $\beta$ are in $\mathbf{\Lambda}_{a, p}^{\alpha-\beta}$.

The multiplicity of indices may seem to be an annoyance, but they are really held to a minimum necessary for precise statements. Indeed, for Sobolev's Lemma and Bernstein's Theorem it is desirable to complicate the Lebesgue spaces. Sobolev's Lemma in the form $\mathbf{\Lambda} \subset \mathrm{L}_{a}$ (which includes the original

\footnotetext{
* Part of the research for this paper was sponsored by the National Science Foundation Contracts GP 6145 and 7478.
} 
Sobolev statement) is given as Theorem 4 in $\S 8$. If, however, we introduce the Lorentz spaces $\mathbf{L}_{a, p}$ we get the more accurate

Theorem 5. If $1 / a-\alpha / n=1 / b>0$ and $\alpha>0$ then $\mathbf{\Lambda}_{a, p}^{\alpha} \subset \mathbf{L}_{b, p}$.

The Lorentz spaces $\mathbf{L}_{a, p}$ are defined for $1<a<\infty$ in such a way that $\mathbf{L}_{a, p}$ increases as a function of $p, 1 \leqq p \leqq \infty$, and $\mathrm{L}_{a, a}=\mathrm{L}_{a}$. The precise definition is given at the end of this introduction. (In case $1 / a-\alpha / n=0$ the statement of Theorem 5 is replaced by $\mathbf{\Lambda}_{a, 1}^{\alpha} \subset \mathbf{C}_{0}$ ).

It is even more vital to complicate the Lebesgue spaces for the purposes of Bernstein's Theorem. In $\S 2$ we define spaces ${ }_{a} L_{p}$ such that for fixed $p, 1 \leqq p \leqq \infty$, ${ }_{a} \mathrm{~L}_{p}$ is a decreasing function of $a$ in $1 \leqq a \leqq \infty$, and ${ }_{p} \mathrm{~L}_{p}=\mathrm{L}_{p}$. The sharp $n$ dimensional Bernstein Theorem is given in $\S 3$ as

Theorem 1. For $1 \leqq a \leqq 2$ and $\alpha=n / p-n / a^{\prime}$ we have $\mathcal{F}: \mathbf{\Lambda}_{a, p}^{\alpha} \rightarrow{ }_{a}{ }^{\prime} \mathbf{L}_{p}$.

This is our main result. A corollary is that

For $\alpha=n / p-n / 2$ the Fourier transform gives an isomorphism of $\mathbf{\Lambda}_{2, p}^{\alpha}$ with ${ }_{2} \mathbf{L}_{2}$.

The classical Bernstein Theorem had evolved to the point where the statement $\mathcal{F}: \mathbf{\Lambda}_{2,1}^{n / 2} \rightarrow \mathbf{L}_{1}$ was, more or less, known. The spaces $\boldsymbol{\Lambda}_{2,1}^{n / 2}$ are Banach algebras under ordinary multiplication of functions and might well be called the Bernstein algebras. A decisive step was taken by Beurling [1] who proved, but did not state, the case $n=1$ of $\mathcal{F}\left(\Lambda_{2,1}^{n / 2}\right)={ }_{2} \mathrm{~L}_{1}$ by precisely identifying the convolution-subalgebra of $\mathrm{L}_{1}$, namely ${ }_{2} \mathrm{~L}_{1}$, corresponding to the Bernstein algebra.

The ${ }_{a} \mathbf{L}_{p}$ spaces and the related $\mathbf{K}_{a, p}$ spaces, which for fixed a increase with $p$ and have $\mathbf{K}_{a, a}=\mathbf{L}_{a}$, are taken up in $\$ 2$. Proposition 2.4 is the key to Theorem 1 .

The Fourier transform is studied in $\$ 3$. Our treatment unifies a considerable amount of the classical theory. In addition to Theorem 1 there are three statements about Fourier transforms of $\mathbf{L}_{p}$ worth singling out:

(a) $\mathcal{F}: \mathrm{L}_{p} \rightarrow \mathrm{L}_{p^{\prime}, p}$ for $1<p \leqq 2$,

(b) $\mathfrak{F}: \mathbf{L}_{p} \rightarrow \mathbf{K}_{p^{\prime}, 2}$ for $1<p \leqq 2$,

(c) $\mathcal{F}: \mathbf{L}_{p} \rightarrow \mathbf{\Lambda}_{p^{\prime}, p}^{0}$ for $1 \leqq p \leqq 2$.

Each of these statements is independent of the others. The assertion (a) is the classical Hausdorff-Young-Paley Theorem. Statement (b) is new, and it is the underlying fact for the theory of lacunary Fourier transforms. The content of (c) is mysterious, and we shall discuss it further shortly. At any rate, these three results seem to contain all that is known about $\mathbf{L}_{p}$ Fourier transforms for $1<p \leqq 2$.

Theorem 2 , in $\$ 4$, gives a formula for inverting difference operators. This opens the way for Theorem 3 , the basic inclusion relation among Lipschitz spaces given in $\$ 5$, and a variety of equivalent methods for defining the $\mathbf{\Lambda}_{a, p}^{\alpha}$ norm. The $\boldsymbol{\Lambda}_{a, p}^{\alpha}$ norm is particularly well-suited to the Riesz potentials, taken up 
in §6. If $R^{\beta}$ signifies the Riesz potential of order $\beta$, then $R^{\beta} \boldsymbol{\Lambda}_{a, p}^{\alpha}=\boldsymbol{\Lambda}_{a, p}^{\alpha+\beta}$. Having various methods for defining the $\boldsymbol{\Lambda}_{a, \nu}^{\alpha}$-norm, we can extend the definition of the Lipschitz spaces to $\boldsymbol{\Lambda}_{a, p}^{\alpha}$ with $\alpha \leqq 0$. We are also able, in $\S 7$, to obtain duality and interpolation theorems. The last section, $\S 8$, is devoted to Theorem 4 and 5 which are generalizations of Sobolev's Lemma.

We tend to state our results in the form $X \subset Y$ or $\mathcal{F}: X \rightarrow Y$ where $X$ and $Y$ are Banach spaces. Nearly all statements are best possible. For the most part we have eschewed concrete counter-examples; instead general arguments requiring little, if any, calculation are used.

There is, aside from minor details, only one important problem in the subject which remains open. We prove in Proposition 1.6 that, for $0<\alpha \leqq n$, the space $\boldsymbol{\Lambda}_{n / \alpha, 1}^{\alpha}$ is a subalgebra of $\mathbf{C}_{0}$. The Bernstein Theorem states that $\boldsymbol{\Lambda}_{n / \alpha, 1}^{\alpha} \subset \mathbf{A}$, the Fourier algebra, if $n / 2 \leqq \alpha \leqq n$. These Lipschitz algebras are treated in [3]. What remains unexamined is the limiting case $\alpha=0$. We know that $\mathbf{A} \subset \mathbf{\Lambda}_{\infty, 1}^{0} \subset \mathbf{C}_{0}$, the inclusion being proper, and that $\mathbf{\Lambda}_{\infty, 1}^{0}$ is invariant under affine transformations of $R^{n}$. Is $\boldsymbol{\Lambda}_{\infty, 1}^{0}$ a Banach algebra? If it is, what is the functional calculus in $\boldsymbol{\Lambda}_{\infty, 1}^{0}$ ? (Lipschitz functions operate on $\boldsymbol{\Lambda}_{n / \alpha, 1}^{\alpha}$ when $0<\alpha<1$ ).

In the theory of Fourier series, rather than Fourier integrals, there is no question of behavior at $\infty$ in the Lipschitz spaces. The theory would then be somewhat simpler, but it is doubtful that any real advantages would accrue. The correspondences to the classical Lipschitz spaces used in Fourier series with our spaces are, for $0<\alpha<1$,

$$
\begin{aligned}
& \mathbf{\Lambda}_{\infty, \infty}^{\alpha} \leftrightarrow \operatorname{lip}^{\alpha}=\lambda^{\alpha} \\
& \mathbf{\Lambda}_{\infty, \infty}^{1} \leftrightarrow \lambda^{*} \\
& \mathbf{\Lambda}_{a, \infty}^{\alpha} \leftrightarrow \lambda_{a}^{\alpha} \\
& \mathbf{\Lambda}_{a, \infty}^{1} \leftrightarrow \lambda_{a}^{*} .
\end{aligned}
$$

\section{Preliminary notations.}

$d x=$ Lebesgue measure on $R^{n}$.

$\nexists h=$ dilation invariant measure on $R^{n}-\{0\}$ conventionally normalized as $\pi h=d h / n v(h)$ where

$v(h)=b_{n}|h|^{n}=$ volume of the ball of radius $|h|$. We also write

$d t=t^{-1} d t=$ normalized Haar measure on the multiplicative group of positive real numbers.

Note that with the above normalizations, if $u(h)=\varphi(|h|)$ then

$$
\int u(h) d h=\int \varphi(t) d t .
$$

In $\S 2^{*}$ we use $d h$ to mean $v^{-1}(h) d h$ so that if $u(h)=\varphi[v(h)]$ then $\int u(h)$ dh $=$ $\int \varphi(v) d v$. 
$\mathrm{L}_{a}, \mathbf{1} \leqq a \leqq \infty$, is the Lebesgue space on $R^{n}$ with norm

$$
\|f\|_{a}=\left\{\int|f(x)|^{a} d x\right\}^{1 / a}
$$

$\mathfrak{L}_{p}, 1 \leqq p \leqq \infty$, is, according to the context, the Lebesgue space on $R^{n}-\{0\}$ or $(0, \infty)$ for the dilation invariant measure. The norm is

$$
\|u\|_{p}=\left\{\int|u(h)|^{p} d h\right\}^{1 / p} .
$$

$\mathfrak{L}_{p}(\mathrm{X})$, for $\mathrm{X}$ a Banach space, is the Banach space of $\mathrm{X}$-valued functions on $R^{n}-\{0\}$ or $(0, \infty)$ with the norm

$$
\|u\|_{X, p}=\left\{\int(X[u(h)])^{p} d h\right\}^{1 / p}
$$

where $X(f)$ is the norm of $f \varepsilon \mathrm{X}$. In particular, $\mathfrak{L}_{p}\left(\mathrm{~L}_{a}\right)$ consists of functions $u(x ; h)$ with finite norm

$$
\|u\|_{a, p}=\left\{\int\|u(\cdot ; h)\|_{a}^{p} d h\right\}^{1 / p}
$$

Conjugate indices are designated by a prime: $a^{\prime}=a /(a-1)$.

$D=$ space of infinitely differentiable functions of compact support on $R^{n}$.

$\mathcal{O}=$ subspace of $D$ constituted by the functions with support disjoint from the origin.

$\mathcal{S}=$ space of functions $f$ on $R^{n}$ such that $P(x) Q(\partial / \partial x) f$ is bounded and continuous for all polynomials $P$ and $Q$.

For $1 \leqq a<\infty$, the completion of $\mathscr{D}$ or $S$ for the $\mathbf{L}_{a}$-norm is $\mathbf{L}_{a}$.

$\mathbf{C}_{0}=$ completion of $S$ or $D$ for the $\mathbf{L}_{\infty}$-norm.

$\mathbf{L}_{a, p}$ is the Lorentz space defined below.

${ }_{a} \mathbf{L}_{p}$ is defined in $\$ 2$.

$\mathbf{\Lambda}_{a, p}^{\alpha}$ is defined for $\alpha>0$ in $\S 1$ and for $\alpha \leqq 0$ in $\S 6$.

$\mathbf{K}_{a, p}^{\alpha}$ and $\mathbf{K}_{a, p}=\mathbf{K}_{a, p}^{0}$ are defined in $\S 2$.

The Fourier transform is

$$
\hat{f}(\xi)=\int e^{-2 \pi i \xi x} f(x) d x .
$$

Lorentz spaces. Given a measurable function $f$ one defines a decreasing function $f^{*}$ on $(0, \infty)$ by

$$
f^{*}(v)=\sup _{E} \inf _{x \varepsilon E}|f(x)|
$$

where $E$ runs through the sets of measure $\geqq v$. The function $f^{*}$ is called the decreasing rearrangement of $f$. If $f$ is integrable over sets of finite measure we can form

$$
f^{* *}(t)=t^{-1} \int_{0}^{t} f^{*}(v) d v
$$


The mapping $f \rightarrow f^{* *}$ is sublinear, i.e. $(f+g)^{* *} \leqq f^{* *}+g^{* *}$ and $(c f)^{* *}=$ $|c| f^{* *}$. Hence, if $M$ is a norm for functions on $(0, \infty)$ which is without structure, i.e. $|\varphi| \leqq|\psi|$ implies $M(\varphi) \leqq M(\psi)$, then $M^{* *}(f)=M\left(f^{* *}\right)$ defines a norm on measurable functions which are integrable over sets of finite measure. For $1<a<\infty$ and $1 \leqq p \leqq \infty$ the Lorentz $\mathrm{L}_{a, p}$ norm is defined as

$$
\|f\|_{a, p}=\left\|f^{* *} v^{1 / a}\right\|_{p}=\left\{\int_{0}^{\infty}\left[f^{* *}(v) v^{1 / a}\right]^{p} d v\right\}^{1 / p} \text {. }
$$

Since $f \rightarrow f^{*}$ is not sublinear, the quantity $M^{*}(f)=M\left(f^{*}\right)$ does not yield a norm in general. On the other hand, $f^{*} \leqq f^{* *}$; so $M\left(f^{*}\right) \leqq M\left(f^{* *}\right)$ always. In the case of the Lorentz norms, the Hardy inequality gives

$$
\left\|f^{* *} v^{1 / a}\right\|_{p} \leqq a^{\prime}\left\|f^{*} v^{1 / a}\right\|_{p} .
$$

Therefore the Lorentz space $\mathbf{L}_{a, p}$ consists of exactly those measurable functions $f$ for which $\left\|f^{*} v^{1 / a}\right\|_{\nu}$ is finite. The Lorentz spaces are not defined when $a=1$ or $\infty$, for in these cases the formal definition would yield $\|f\|_{a, p}=\infty$ for all non-trivial $f$ and $p<\infty$ while $\|f\|_{1, \infty}=\|f\|_{1}$ and $\|f\|_{\infty, \infty}=\|f\|_{\infty}$. The space of functions for which $f^{*}(v) v$ is bounded is called "weak $L_{1}$ ". It is not a Banach space.

It is easy to prove that $\mathbf{L}_{a, p} \subset \mathbf{L}_{a, q}$ if $p \leqq q$. Since $\left\|f^{*} v^{1 / a}\right\|_{a}=$ $\|f\|_{a}=\left\{\int|f(x)|^{a} d x\right\}^{1 / a}$, the Lebesgue space $\mathbf{L}_{a}$ is the Lorentz space $\mathbf{L}_{a, a}$. The Lorentz spaces are especially convenient for interpolation, see [2; §13.4] and [5]. The dual space of $\mathbf{L}_{a, p}$ is $\mathbf{L}_{a^{\prime}, p^{\prime}}$.

\section{Contents}

1. Lipschitz Spaces (Theorem 0).

2. The spaces ${ }_{a} \mathbf{L}_{p}$ and $\mathbf{K}_{a, p}$.

3. Bernstein's Theorem (Theorem 1).

4. The fundamental inversion formula (Theorem 2).

5. The basic inclusion relations for Lipschitz spaces (Theorem 3).

6. Riesz potentials.

7. Duality and interpolation.

8. Sobolev's Lemma (Theorem 4 and 5).

Note. Proposition 2.4 is the 4 th Proposition of $\$ 2$; Iemma 1.3 is the 3 rd Lemma of $\$ 1$.

1. Lipschitz spaces. A general class of Lipschitz norms for functions on $R^{n}$ is defined this way. For $h \varepsilon R^{n}$ the translation operator $T(h)$ is given by

$$
T(h) f(x)=f(x+h)
$$

and the kth difference operator $\Delta^{k}(h)$ by

$$
\Delta^{k}(h)=\{T(h)-I\}^{k}
$$


where $I$ denotes the identity. Put

$$
u(x ; h)=|h|^{-\alpha} \Delta^{k}(h) f(x) .
$$

Given a translation invariant norm $X$ defined for functions on $R^{n}$ and finite for elements of $S$, we define the Lipschitz norm $\Lambda_{X, p}^{\alpha}$ when $0<\alpha<k$ by

$$
\Lambda_{X, p}^{\alpha}(f)=\|u\|_{X, p} \text {. }
$$

In other words, if we put $\omega(h)=X\left\{\Delta^{k}(h) f\right\}$, then $\Lambda_{X, p}^{\alpha}(f)$ is the $\mathscr{L}_{p}$-norm of $|h|^{-\alpha} \omega(h)$.

We shall only be interested in the $\Lambda_{X, p}^{\alpha}$-norms up to equivalence. The integer $k$ is not too significant in the definition since we have

Lemma 1.1. The norms $\Lambda_{X, p}^{\alpha}$ are, up to equivalence, independent of the choice of $k>\alpha$.

An elementary proof of Lemma 1.1 will be given in the appendix, but the statement is also a by-product of the proof of Theorem 3.

The Lipschitz spaces $\boldsymbol{\Lambda}_{X, p}^{\alpha}$ are defined as the completion of $\hat{\theta}$ for the norm $\Lambda_{X, p}^{\alpha}$. The space $\hat{\mathcal{O}}$ consists of the Fourier transforms of elements of $\mathcal{O}$, the infinitely differentiable functions on $R^{n}$ with compact supports disjoint from the origin (in the periodic analog $\hat{\Theta}$ would be the trigonometric polynomials without constant term).

Henceforth we shall take the $X$-norm to be an $\mathrm{L}_{a}$-norm, $1 \leqq a \leqq \infty$. One might be tempted to consider a norm of the form $X(f)=\sum_{i=1}^{n}\left\|\partial f / \partial x_{i}\right\|_{a}$, but it would turn out that $\mathbf{\Lambda}_{X, p}^{\alpha}=\mathbf{\Lambda}_{a, p}^{\alpha+1}$.

The character of the Lipschitz spaces $\mathbf{\Lambda}_{a, p}^{\alpha}$ depends critically on the degree of smoothness, $\alpha-n / a$. The importance of this quantity is a consequence of the next simple fact.

Given a function $f$ defined on $R^{n}$ and $t>0$ we put

$$
f^{t}(x)=f(t x), \quad f_{t}(x)=t^{-n} f\left(t^{-1} x\right) .
$$

Lemma 1.2. $\Lambda_{a, p}^{\alpha}\left(f^{t}\right)=t^{\alpha-n / a} \Lambda_{a, p}^{\alpha}(f)$ and $\Lambda_{a, p}^{\alpha}\left(f_{t}\right)=t^{-\alpha-n / a^{\prime}} \Lambda_{a, p}^{\alpha}(f)$.

Proof. Elementary calculation.

The Lipschitz spaces $\mathbf{\Lambda}_{a, p}^{\alpha}$ were defined as completions of $\hat{\mathcal{O}}$. This is a technical convenience which will be especially useful in many proofs. The definition does not give much insight into the nature of Lipschitz spaces. To remedy this defect we shall consider the relation of $\mathbf{\Lambda}_{a, p}^{\alpha}$ to $D L_{1}$ and $D^{\prime} C_{0}$, where $D L_{1}$ consists of the infinitely differentiable functions all of whose derivatives belong to $\mathbf{L}_{\mathbf{1}}$ and $D^{\prime} C_{0}$ is the closure of $D$ in the dual space of $D L_{1} ;$ more specifically: $f \varepsilon D^{\prime} C_{0}$ iff $f$ represents a distribution such that $f * u \varepsilon \mathbf{C}_{0}$ for each $u \varepsilon D$.

Proposition 1.1. $D L_{1}$ is a subspace of and $D$ is dense in each $\mathbf{\Lambda}_{a, p}^{\alpha}$ with $\alpha>0$.

Proof. Let $K(f)$ denote the sum of the $\mathbf{L}_{1}$-norms of all derivatives of $f$ up to a suitably large order depending on an integer $k$ and the dimension $n$. Besides the trivial inequalities

$$
\|f\|_{1} \leqq K(f), \quad\left\|\Delta^{k}(h) f\right\|_{1} \leqq k ! K(f)|h|^{k}
$$


one has also

These yield

$$
\|f\|_{\infty} \leqq c K(f),\left\|\Delta^{k}(h) f\right\|_{\infty} \leqq k ! c K(f)|h|^{k}
$$

$$
|h|^{-\alpha}\left\|\Delta^{k}(h) f\right\|_{a} \leqq \text { const. } K(f) \min \left\{|h|^{k-\alpha},|h|^{-\alpha}\right\}
$$

for all $a, 1 \leqq a \leqq \infty$. Thus $\Lambda_{a, p}^{\alpha}(f) \leqq$ const. $K(f)$. Let $\overline{\mathbf{\Lambda}}_{a, p}^{\alpha}$ denote the completion of $D L_{1}$ for the $\Lambda_{a, p}^{\alpha}$-norm. Since $\hat{\Theta} \subset D L_{1}$, there is the obvious inclusion $\mathbf{\Lambda}_{a, p}^{\alpha} \subset \overline{\boldsymbol{\Lambda}}_{a, p}^{\alpha}$. What has to be proved is that if $f \rightarrow \int f T$ gives a bounded linear functional on $\overline{\mathbf{\Lambda}}_{a, p}^{\alpha}$ such that $\int f T=0$ for all $f \varepsilon \hat{\mathcal{O}}$ then $\int f T=0$ for all $f \varepsilon \mathfrak{D} L_{1}$. The $T$ in question may be viewed as a tempered distribution. To say that $T$ is orthogonal to $\hat{\Theta}$ is to say that $\hat{T}$ is orthogonal to $\mathcal{O}$. This means that the support of the distribution $\hat{T}$ is the origin, i.e. $T$ is a polynomial. Now the only polynomials which give continuous functionals on $D L_{1}$ are the constants. Hence $\int f T=c \int f(x) d x$ for some constant $c$. If this functional is bounded in the $\Lambda_{a, p^{-}}^{\alpha}$ norm then for some $M$,

$$
\left|c \int f(x) d x\right| \leqq M \Lambda_{a, p}^{\alpha}(f) .
$$

Using Lemma 1.2 we have for all $t>0$

$$
\left|c \int f(x) d x\right|=\left|c \int f_{t}(x) d x\right| \leqq M \Lambda_{a, p}^{\alpha}\left(f_{t}\right)=t^{-\alpha-n / a^{\prime}} M \Lambda_{a, p}^{\alpha}(f),
$$

and so $c \int f(x) d x=0$ for all $f \varepsilon D L_{1}$. This proves that $D L_{1}$ is a dense subspace of $\mathbf{\Lambda}_{a, p}^{\alpha}$, but since $D$ is dense in $D L_{1}$ for the $K$ norm, $D$ is also dense in $\mathbf{\Lambda}_{a, p}^{\alpha}$.

The next is a sort of dual statement to Proposition 1.1. Write $\left[D^{\prime} C_{0}\right]_{N}$ for the space of distributions whose $N$ th order partial derivatives are in $D^{\prime} C_{0}$ modulo the space of polynomials of degree $\leqq N$.

Proposition 1.2. (i) $\mathbf{\Lambda}_{a, 1}^{n / a} \subset \mathrm{C}_{0}$.

(ii) $\mathbf{\Lambda}_{a, p}^{\alpha} \subset D^{\prime} C_{0}$ if $\alpha<n / a$.

(iii) If $N$ is the greatest integer such that

$$
N \leqq \alpha-n / a(N<\alpha-n / a \text { if } p=1) \text { then } \mathbf{\Lambda}_{a, p}^{\alpha} \subset\left[D^{\prime} C_{0}\right]_{N} .
$$

The proof of Proposition 1.2 (i) will be given after Theorem 2. The other two statements will be seen to be corollaries of the first. If $\beta=\alpha-n / a$ then, by Theorem 3 below, $\mathbf{\Lambda}_{a, p}^{\alpha} \subset \mathbf{\Lambda}_{\infty, \infty}^{\beta}$, and for $f \varepsilon \mathbf{\Lambda}_{\infty, \infty}^{\beta}$ with $\beta>0$ we have $\left\|\Delta^{k}(h) f\right\|_{\infty}=$ $O\left(|h|^{\beta}\right)$ when $k>\beta$ as $|h| \rightarrow 0$, $\infty$. Part of the assertion of Proposition 1.2 (iii) states, however, that polynomials of degree $\leqq N$ represent the null element of $\mathbf{\Lambda}_{a, p}^{\alpha}$. Thus $\mathbf{\Lambda}_{a, p}^{\alpha}$ is not, for $N \geqq 0$, a space of distributions although any functionrepresentative of an element is continuous if $\alpha>n / a$. By contrast we have

Remark. If $\alpha<n / a$ or $\alpha=n / a$ and $p>1$ then $\mathbf{\Lambda}_{a, p}^{\alpha}$ contains a function of compact support which is infinitely differentiable except at the origin where $\lim _{x \rightarrow 0} f(x)=+\infty$. 
Both the remark and the fact that polynomials of degree $\leqq N$ represent the null element of $\boldsymbol{\Lambda}_{a, p}^{\alpha}$ are proved by taking a fixed $u \varepsilon \mathfrak{D}$ which is identically 1 on a neighborhood of the origin. If $\alpha<n / a$ then $\lim t \rightarrow \infty \Lambda_{a, p}^{\alpha}\left(u^{t}\right)=0$ by Lemma 1.2. Hence there exists a sequence $\left\{c_{j}\right\}$ of positive constants such that $\sum c_{j} \Lambda_{a, p}^{\alpha}\left(u^{j}\right)<\infty$ but $\sum c_{i}=\infty$. Put $f(x)=\sum c_{i} u^{i}(x)=\sum c_{i} u(j x)$; for each $x \neq 0$ the sum is finite. Obviously $f \varepsilon \mathbf{\Lambda}_{a, p}^{\alpha}$, but $f(0)=\sum c_{j}=+\infty$. This proves the remark in case $\alpha<n / a$; if $\alpha=n / a$ and $p>1$ we need a slight modification. Put $v^{(j)}=j^{-1} \sum_{t=1}^{i} u^{t}$. Then

$$
|h|^{-\alpha}\left\|\Delta^{k}(h) v^{(i)}\right\|_{a} \leqq j^{-1} \sum_{t=1}^{i} \varphi(t h)
$$

where $\varphi(h)=|h|^{-\alpha}\left\|\Delta^{k}(h) u\right\|_{a}$. To say that $u \varepsilon \mathbf{\Lambda}_{a, p}^{\alpha}$ is to say that $\varphi \varepsilon \mathfrak{L}_{p}$ for $p<\infty$ or $\varphi \varepsilon \mathfrak{C}_{0}$ if $p=\infty$ with $\|\varphi\|_{p}=\Lambda_{a, p}^{\alpha}(u)$. For any $\varphi \varepsilon \mathfrak{L}_{p}, 1<p<\infty$, or $\varphi \varepsilon \mathcal{C}_{0}$ we have $\lim _{j \rightarrow \infty} j^{-1} \sum_{t=1}^{i} \varphi^{t}=0$ in the norm of $\mathcal{L}_{p}$, i.e. $\lim _{i \rightarrow \infty} \Lambda_{a, p}^{\alpha}\left(v^{(i)}\right)=0$ for $p>1$. We put $f(x)=\sum c_{j} v^{(i)}(x)$, and obtain the same conclusion as before. Now suppose that $P$ is a homogeneous polynomial of degree $d \leqq \alpha-n / a$. Then $\lim _{t \rightarrow 0} P u^{t}=P$ uniformly on compact sets, but $P u^{t}=t^{-d}(P u)^{t}$; so, by Lemma 1.2 ,

$$
\Lambda_{a, p}^{\alpha}\left(P u^{t}\right)=t^{-d} t^{\alpha-n / a} \Lambda_{a, p}^{\alpha}(P u) \rightarrow 0
$$

as $t \rightarrow 0$ if $d<\alpha-n / a$. In case $d=N=\alpha-n / a$ (so $p>1$ ) we consider the sequence $\left\{P w^{(i)}\right\}$ where $w^{(i)}=j^{-1} \sum_{i=1}^{i} u^{1 / i}$. As before, $\lim _{i \rightarrow \infty} \Lambda_{a, p}^{\alpha}\left(P w^{(j)}\right)=0$, but $\lim _{j \rightarrow \infty} P w^{(i)}=P$.

The fact that the Lipschitz spaces are not always spaces of true distributions is a triviality. Let $S_{*}^{\prime}$ signify the space of restrictions of tempered distributions to $R^{n}-\{0\}$. The Fourier transform space is $s^{\prime} / P$, tempered distributions modulo polynomials, and we have

Corollary. For all $\alpha, a, p$ there is a continuous injection $\mathbf{\Lambda}_{a, p}^{\alpha} \subset \mathcal{S}^{\prime} / \mathcal{\odot}$.

The simplicity of the definition of the $\Lambda_{a, p}^{\alpha}$-norm might appear to be marred by the perhaps annoying technicality that the space $\boldsymbol{\Lambda}_{a, p}^{\alpha}$ is always defined as the completion of $\hat{\mathcal{O}}$ for this norm. There is no real problem, however, whenever one has to do with reasonable functions. The next is for its own interest, it is not needed, in the generality stated, anywhere in this work.

Theorem 0. Let $f$ be a measurable function representing an element of $D^{\prime} C_{0}$. If $\Lambda_{a, p}^{\alpha}(f)<\infty$ (where $\left.\alpha>0\right)$ then $f \varepsilon \mathbf{\Lambda}_{a, p}^{\alpha}$ when $1 \leqq a<\infty, 1 \leqq p<\infty$. The statement holds with $p=\infty$ if, for some $k>\alpha,\left\|\Delta^{k}(h) f\right\|_{a}=o\left(|h|^{\alpha}\right)$ as $|h| \rightarrow 0$ and $|h| \rightarrow \infty$; it remains valid for $a=\infty$ provided that for each $h, \Delta^{k}(h) f \varepsilon \mathbf{C}_{0}$.

Proof. Take $w \varepsilon$ D such that $w \geqq 0$ and $\int w(x) d x=1$. Then for each $t>0$ and each $h$ we have

$$
\left\|\Delta^{k}(h)\left(w_{t} * f\right)\right\|_{a}=\left\|w_{t} * \Delta^{k}(h) f\right\|_{a} \leqq\left\|\Delta^{k}(h) f\right\|_{a}
$$


and also

$$
\lim _{t \rightarrow 0}\left\|\Delta^{k}(h)\left(w_{t} * f-f\right)\right\|_{a}=\lim _{t \rightarrow 0}\left\|w_{t} * \Delta^{k}(h) f-\Delta^{k}(h) f\right\|_{a}=0 .
$$

Hence, by dominated convergence, $\lim _{t \rightarrow 0} \Lambda_{a, p}^{\alpha}\left(w_{t} * f-f\right)=0$. Therefore the result follows if $w_{t} * f \varepsilon \mathbf{\Lambda}_{a, p}^{\alpha}$ for all small $t$. Since $w_{t} * f \varepsilon D C_{0}$ whenever $f \varepsilon D^{\prime} C_{0}$, it suffices to prove the theorem under the stronger hypothesis that $f \varepsilon D C_{0}$. (The proof is completed by Lemmas 1.7 and 1.8 at the end of this section.)

Suppose $0<\alpha<1$. Let $F$ be an infinitely differentiable function on the complex plane such that

$$
\begin{aligned}
& F(z)=z \text { for }|z| \geqq 1, F(z)=0 \text { for }|z|<\frac{1}{2}, \\
& \quad \text { and }|F(z)-F(\zeta)| \leqq c|z-\zeta| \text { for some constant } c \text { and all } z, \zeta .
\end{aligned}
$$

Put $F_{\epsilon}(z)=\epsilon F\left(\epsilon^{-1} z\right)$. Now suppose $f \varepsilon D C_{0}$ and $\Lambda_{a, p}^{\alpha}(f)<\infty$. Then $F_{\epsilon}(f) \varepsilon \mathbb{D}$ for each $\epsilon>0$; in particular $F_{\epsilon}(f) \varepsilon \mathbf{\Lambda}_{a, p}^{\alpha}$. On the other hand

$$
\left|\Delta(h)\left\{f-F_{\epsilon}(f)\right\}\right| \leqq(1+c)|\Delta(h) f| \text { and } \lim _{\epsilon \rightarrow 0} F_{\epsilon}(f)-f=0 .
$$

It follows by dominated convergence that $\lim _{\epsilon \rightarrow 0} \Lambda_{a, p}^{\alpha}\left\{F_{\epsilon}(f)-f\right\}=0$. This proves Theorem 0 when $0<\alpha<1$.

Unfortunately, the above argument depends on the use of a first difference. It does not seem possible to modify this reasoning to treat $\alpha \geqq 1$. When $\alpha \geqq n / a$, Theorem 0 follows from the straightforward Lemma 1.7. Thus, when $n=1$, Theorem 0 is entirely elementary. When $n>1$, we have to use the sophisticated Lemma 1.8. Let it be said that the difficulty in Theorem 0 comes about because, when $\alpha<n / a$, the $a$ priori estimate for the size of $f$ at $\infty$ is inadequate. Had we assumed $f \varepsilon \mathrm{L}_{b}$ for any $b, 1 \leqq b \leqq \infty$, such that $1 / a-\alpha / n \leqq 1 / b$ then Lemma 1.8 could be replaced by a modification of Lemma 1.7.

Returning to the Lipschitz spaces defined as completions of $\hat{\mathcal{O}}$, using the Corollary to Proposition 1.2, and noting that the convolution of an element of $\hat{\mathcal{O}}$ with an element of $\mathcal{S}^{\prime} / \mathcal{P}$ is a well-defined continuous function, we see that the next makes perfect sense

Proposition 1.3. $\mathbf{\Lambda}_{a, p}^{\alpha} * \mathbf{\Lambda}_{b, q}^{\beta} \subset \mathbf{\Lambda}_{c, r}^{\alpha+\beta}$ whenever $1 / c=1 / a+1 / b-1$ and $1 / r=1 / p+1 / q$.

Proof. Take $f, g \varepsilon \hat{\mathcal{O}}$ and choose $k>\alpha, l>\beta$. According to Lemma 1.1 we may define the $\Lambda_{a, y}^{\alpha}$-norm of $f$ using a $k^{\text {th }}$ difference, the $\Lambda_{b, q}^{\beta}$-norm of $g$ using an $l^{\text {th }}$ difference and the $\Lambda_{c, r}^{\alpha+\beta}$-norm of $f * g$ using a $(k+l)^{\text {th }}$ difference. Since

$$
|h|^{-\alpha-\beta} \Delta^{k+l}(h) f * g=|h|^{-\alpha} \Delta^{k}(h) f *|h|^{-\beta} \Delta^{l}(h) g
$$

and $\|u * v\|_{c} \leqq\|u\|_{a}\|v\|_{b}$ when $1 / c=1 / a+1 / b-1$, we have

$$
|h|^{-\alpha-\beta}\left\|\Delta^{k+l}(h) f * g\right\|_{c} \leqq \varphi(h) \psi(h),
$$


where the $\mathfrak{L}_{p}$-norm of $\varphi$ is $\Lambda_{a, p}^{\alpha}(f)$ and the $\mathfrak{L}_{q}$-norm of $\psi$ is $\Lambda_{b, q}^{\beta}(g)$. Since $\|\varphi \psi\|_{r} \leqq$ $\|\varphi\|_{p}\|\psi\|_{a}$ when $1 / r=1 / p+1 / q$ we arrive at $\Lambda_{c, r}^{\alpha+\beta}(f * g) \leqq \Lambda_{a, p}^{\alpha}(f) \Lambda_{b, q}^{\beta}(g)$, and this proves the proposition.

It is worthwhile noting at this point that statement (ii) of Proposition 1.2 is a corollary of statement (i). If $f \varepsilon \mathbf{\Lambda}_{a, p}^{\alpha}, u \varepsilon \mathfrak{D}$, and $\alpha<n / a$ then, by Proposition 1.1, we have $u \varepsilon \Lambda_{1, p^{\prime}}^{\beta}$ where $\beta=n / a=\alpha$. It follows from Proposition 1.3 that $f * u \varepsilon \mathbf{\Lambda}_{a, 1}^{n / a}$. Thus $f * u \varepsilon \mathbf{C}_{0}$, and, since this holds for each $u \varepsilon \mathbb{D}, f \varepsilon \mathbb{D}^{\prime} C_{0}$. Statement (iii) is deduced from (ii) by observing (see $\$ 6$ ) that if $f \varepsilon \mathbf{\Lambda}_{a, p}^{\alpha}$ then its first derivatives belong to $\mathbf{\Lambda}_{a, p}^{\alpha-1}$.

It is generally useful to know the effect of smoothing operations.

Lemma 1.4. Suppose $w \varepsilon \mathrm{L}_{1}$; then for each $t>0$ convolution with $w_{t}$ maps $\mathbf{\Lambda}_{a, p}^{\alpha}$ continuously into itself and

(i) if $\int w(x) d x=1$ then $w_{t} *$ tends strongly to the identity as $t \rightarrow 0$,

(ii) $w_{t} *$ tends strongly to the zero-operator as $t \rightarrow \infty$.

Proof. Since $\Delta^{k}(h)(w * f)=w * \Delta^{k}(h) f$ we get

and hence

$$
\left\|\Delta^{k}(h)(w * f)\right\|_{a} \leqq\|w\|_{1}\left\|\Delta^{k}(h) f\right\|_{a},
$$

$$
\Lambda_{a, p}^{\alpha}(w * f) \leqq\|w\|_{1} \Lambda_{a, p}^{\alpha}(f) .
$$

Now, given $w \varepsilon \mathrm{L}_{1}$ and $\epsilon>0$ there exists $v \varepsilon S$ and $\delta>0$ such that

$$
\|w-v\|_{1}<\epsilon, \quad \hat{v}(\xi)=\hat{w}(0) \text { for }|\xi|<\delta, \quad \hat{v}(\xi)=0 \text { for } \quad|\xi|>\delta^{-1} \text {. }
$$

It follows that $\Lambda_{a, p}^{\alpha}\left(w_{t} * f-v_{t} * f\right)<\epsilon \Lambda_{a, p}^{\alpha}(f)$ for all $f \varepsilon \hat{\mathcal{O}}$ and $t>0$. Since $\hat{\mathcal{O}}$ is a convolution ideal of $S$, we have $v_{t} * f \varepsilon \hat{\theta}$ which allows us to conclude that $w_{t} *$ maps $\mathbf{\Lambda}_{a, p}^{\alpha}$ into itself. Also, given $f \varepsilon \hat{\mathcal{O}}$ there exists $\delta(f)>0$ such that $\hat{f}(\xi)=0$ when $|\xi|<\delta(f)$ and when $|\xi|>\delta^{-1}(f)$. Hence $v_{t} * f=0$ for $t>\delta^{-1} \cdot \delta^{-1}(f)$ (this proves (ii) and $v_{t} * f=\hat{w}(0) f$ for $t<\delta \cdot \delta(f)$ (this proves (i)).

Let $\mathbf{L}_{b}^{(0)}$ signify the completion of $\hat{\theta}$ for the $\mathbf{L}_{b}$-norm (so $\mathbf{L}_{b}^{(0)}=\mathbf{L}_{b}$ for $1<$ $b<\infty, L_{\infty}^{(0)}=\mathbf{C}_{0}$, and $\mathbf{L}_{1}^{(0)}$ consists of the $f \varepsilon \mathbf{L}_{1}$ with $\left.\int f(x) d x=0\right)$. Then $\mathbf{\Lambda}_{a, p}^{\alpha}$ and $\mathbf{L}_{b}^{(0)}$ are both completions of $\hat{\mathcal{\theta}}$, and they are both subspaces of $\boldsymbol{s}^{\prime} / \mathcal{P}$. What is not altogether trivial is that the intersection (as subspaces of $s^{\prime} / \odot$ ) is the completion of $\hat{\mathcal{O}}$ for the norm $\Lambda_{a, p}^{\alpha}(\cdot)+\|\cdot\|_{b}$.

Proposition 1.4. $\hat{\mathcal{O}}$ is dense in $\mathbf{\Lambda}_{a, p}^{\alpha} \cap \mathrm{L}_{b}^{(0)}$.

Proof. Let $f \varepsilon \mathbf{\Lambda}_{a, p}^{\alpha} \cap \mathbf{L}_{b}^{(0)}$ and $\epsilon>0$ be given. We shall find $g \varepsilon \hat{\mathcal{O}}$ such that $\Lambda_{a, p}^{\alpha}(f-g)<\epsilon$. To do this choose $w \varepsilon S$ such that $\hat{w}=1$ on a neighborhood of the origin, and then choose $t>0$ so that

$$
\begin{aligned}
& \Lambda_{a, p}^{\alpha}\left(f-w_{t} * f\right)<\epsilon / 3, \quad\left\|f-w_{t} * f\right\|_{b}<\epsilon / 3 \\
& \Lambda_{a, p}^{\alpha}\left(w_{1 / t} * f\right)<\epsilon / 3, \quad\left\|w_{1 / t} * f\right\|_{b}<\epsilon / 3 .
\end{aligned}
$$

The function $g$ will have the form $g=\left(w_{t}-w_{1 / t}\right) * \tilde{g}$ where $\tilde{g} \varepsilon \hat{\mathcal{\theta}}$. We may write

$$
f-g=\left(f-w_{t} * f\right)+\left(w_{1 / t} * f\right)+\left(w_{t}-w_{1 / t}\right) *(f-\tilde{g}) .
$$


The proposition is proved once $\tilde{g}$ is picked so that both $\Lambda_{a, p}^{\alpha}(v)<\epsilon / 3$ and $\|v\|_{b}<\epsilon / 3$ where $v=\left(w_{t}-w_{1 / t}\right) *(f-\tilde{g})$. Suppose that $a \geqq b$; then there exists $c$ such that $1 / a=1 / b+1 / c-1$ and $1 \leqq c \leqq \infty$. It is easy to see that $\mathbf{\Lambda}_{c, p}^{\alpha} * \mathbf{L}_{b} \subset \mathbf{\Lambda}_{a, p}^{\alpha}$. Since $w_{t}-w_{1, t} \varepsilon \hat{\Theta}$ the required estimates are obtained once $\|f-\tilde{g}\|_{b}$ is sufficiently small, say $\|f-\tilde{g}\|_{b}<\epsilon /\left\{6\|w\|_{1}+3 \Lambda_{c, p}^{\alpha}\left(w_{t}-w_{1 / t}\right)\right\}$, because $\|v\|_{b} \leqq 2\|w\|_{1}\|f-\tilde{g}\|_{b}$ and $\Lambda_{a, p}^{\alpha}(v) \leqq \Lambda_{c, p}^{\alpha}\left(w_{t}-w_{1 / t}\right)\|f-\tilde{g}\|_{b}$. In case $a<b$ more subtle considerations are required. We appeal to an easy consequence of Theorem 2 (a more general result occurs as Theorem 4) that $\mathbf{\Lambda}_{a, 1}^{\beta} \subset \mathbf{L}_{b}$ if $a<b$ where $\beta=n / a-n / b$. Therefore

$$
\|v\|_{b} \leqq \text { const. } \Lambda_{\alpha, 1}^{\beta}(v) \leqq \text { const. } \Lambda_{1, p^{\prime}}^{\beta-\alpha}\left(w_{t}-w_{1 / t}\right) \Lambda_{a, p}^{\alpha}(f-\tilde{g})
$$

by Proposition 1.3. Hence it suffices to choose $\tilde{g}$ so that $\Lambda_{a, p}^{\alpha}(f-\tilde{g})$ is sufficiently small.

Remark. If $\alpha>0$ then $D$ is dense in $\mathbf{\Lambda}_{a, p}^{\alpha} \cap \mathbf{L}_{1}$.

Convolution operations fit in well with the definition of the $\mathbf{\Lambda}_{a, p}^{\alpha}$-norms. The same is not true for multiplication, although the situation is better than it might at first appear.

Lemma 1.5. Given $\alpha>0, a$, and $p$ there is a constant $c$ such that

$$
\Lambda_{a, p}^{\alpha}(f g) \leqq c\left\{\|f\|_{\infty} \Lambda_{a, p}^{\alpha}(g)+\Lambda_{a, p}^{\alpha}(f)\|g\|_{\infty}\right\}
$$

Proof. There is a Leibnitz formula

$$
\Delta^{k}(h) f g=\sum_{i=0}^{k}\left(\begin{array}{c}
k \\
i
\end{array}\right) \Delta^{i}(h) f \cdot \Delta^{k-i}(h) T^{i}(h) g .
$$

Taking the $\mathbf{L}_{a}$-norms in the space variables we get

$$
\left\|\Delta^{k}(h) f g\right\|_{a} \leqq \sum_{2 i<k}\left(\begin{array}{c}
k \\
i
\end{array}\right) 2^{i}\|f\|_{\infty}\left\|\Delta^{k-i}(h) g\right\|_{a}+\sum_{2 i \geq k}\left(\begin{array}{c}
k \\
i
\end{array}\right) 2^{k-i}\left\|\Delta^{i}(h) f\right\|_{a}\|g\|_{\infty} .
$$

Now suppose $k>2 \alpha$; then all the difference operators $\Delta^{j}(h)$ which occur in the last formula have $j \geqq k / 2>\alpha$. The statement follows from Lemma 1.1

The multiplicative analogue of Proposition 1.3 is one of the deepest results about Lipschitz spaces.

Proposition 1.5. $\quad \mathbf{\Lambda}_{n / \alpha, 1}^{\alpha} \cdot \mathbf{\Lambda}_{b, \alpha}^{\beta} \subset \mathbf{\Lambda}_{b, a}^{\beta}$ whenever $0<\beta<\alpha \leqq n(\beta \leqq \alpha$ if $1 / q \geqq 1 / b-\beta / n)$ and $b<n / \beta(b \leqq n / \beta$ if $q=1)$.

The proof of Proposition 1.5 uses a technical alternative to Lemma 1.5.

Lemma 1.5*. Given $\beta, b, q$ and $p$ such that $1 \leqq b<n / \beta<\infty$ and $1 / p+$ $1 / q \geqq 1 / b$, there is a constant $c$ such that

$$
\Lambda_{b, q}^{\beta}(f g) \leqq c\left\{\|f\|_{\infty}+\Lambda_{B, a}^{\beta}(f)\right\} \Lambda_{b, a}^{\beta}(g)
$$

where $B$ designates the Lorentz $\mathrm{L}_{n / \beta, p}$-norm.

Proof. Define $a$ by $1 / a=1 / b-\beta / n$. Then with $B$ appropriately normalized 
we have $\|\varphi \psi\|_{b} \leqq B(\varphi)\|\psi\|_{a, a}$. Repeating the steps of the proof of Lemma 1.5 we get

$$
\left\|\Delta^{k}(h) f g\right\|_{b} \leqq \sum_{2 i<k}\left(\begin{array}{c}
k \\
i
\end{array}\right) 2^{i}\|f\|_{\infty}\left\|\Delta^{k-i}(h) g\right\|_{b}+\sum_{2 i \geqq k}\left(\begin{array}{c}
k \\
i
\end{array}\right) 2^{k-i} B\left\{\Delta^{i}(h) f\right\}\|g\|_{a, q} .
$$

The previous reasoning, with Lemma 1.1 used both for $X=L_{b}$ and $X=B$, gives

$$
\Lambda_{b, q}^{\beta}(f g) \leqq c\left\{\|f\|_{\infty} \Lambda_{b, q}^{\beta}(g)+\Lambda_{B, q}^{\beta}(f)\|g\|_{a, q}\right\} .
$$

What gives the desired conclusion is the assertion of Theorem 5 that $\|g\|_{a, a} \leqq$ const. $\Lambda_{b, q}^{\beta}(g)$.

Remark. If $1 / q \geqq 1 / b-\beta / n$ one can choose $p=n / \beta$; so $B=L_{n / \beta}$ and all reference to Lorentz spaces disappears.

Proof of Proposition 1.5. Theorem 3 says that $\mathbf{\Lambda}_{n / \alpha, 1}^{\alpha} \subset \mathbf{\Lambda}_{n / \gamma, \alpha}^{\gamma}$ for all $\gamma \leqq \alpha$ and $q \geqq 1$. It follows that $\mathbf{\Lambda}_{n / \alpha, 1}^{\alpha} \subset \mathbf{\Lambda}_{B, q}^{\beta}$ where $B=L_{n / \beta, 1}$ if $\beta<\alpha$ and $B=L_{n / \beta}$ if $\beta=\alpha$. According to Proposition 1.2 (i), $\mathbf{\Lambda}_{n / \alpha, 1}^{\alpha} \subset \mathbf{C}_{0}$. Hence the assertion of the Proposition follows from Lemma 1.5* whenever $b<n / \beta$. When $b=n / \beta$ we have $q=1$; in this case $\mathbf{\Lambda}_{n / \beta, 1}^{\beta} \subset \mathbf{C}_{0}$ and Lemma 1.5 may be used.

Corollary of Proposition 1.5. Under the hypotheses of Proposition 1.5, if $u \varepsilon \mathbf{\Lambda}_{n / \alpha, 1}^{\alpha}$ and $u(0)=1$, then multiplication by $u^{t}$ tends strongly to the identity on $\mathbf{\Lambda}_{b, a}^{\beta}$ as $t \rightarrow 0$.

Proof. Multiplication by $u^{t}$ is bounded in the operator norm by $\Lambda_{n / \alpha, 1}^{\alpha}\left(u^{t}\right)=$ $\Lambda_{n / \alpha, 1}^{\alpha}(u)$. Suppose that $u=1$ on a neighborhood of the origin. Then for each $f \varepsilon \mathbb{D}$ there is a $t(f)>0$ such that $u^{t} f=f$ whenever $0<t<t(f)$. Since $D$ is dense in $\mathbf{\Lambda}_{b, a}^{\beta}$, the assertion of the Corollary is proved under the special supposition about $u$. This supposition is unnecessary because given $u \varepsilon \mathbf{\Lambda}_{n / \alpha, 1}^{\alpha}$ with $u(0)=1$ and $\epsilon>0$ we can find a $v \varepsilon \mathbf{\Lambda}_{n / \alpha, 1}^{\alpha}$ such that $v=1$ on some neighborhood of the origin and $\Lambda_{n / \alpha, 1}^{\alpha}(u-v)<\epsilon$, cf. Proposition 1.7.

Proposition 1.6. Suppose $1 \leqq a \leqq \infty, 1 \leqq p \leqq \infty$. The spaces $\mathbf{\Lambda}_{a, p}^{\alpha} \cap \mathbf{C}_{0}$, for all $\alpha>0$, and the spaces $\mathbf{\Lambda}_{a, p}^{\alpha} \cap \mathbf{L}_{b}$, for $\alpha>n / a$ and $1 \leqq b \leqq \infty$, are regular, self-adjoint, Banach algebras of functions on $R^{n}$. The algebra $D$ is dense in each of these algebras, and, for any $u \varepsilon \mathbb{D}$ such that $u=1$ on a neighborhood of the origin, a sequence $\left\{u^{t}\right\}$ with $t \rightarrow 0$ is an approximate identity in each of these algebras.

Proof. In the case of $\mathbf{\Lambda}_{a, p}^{\alpha} \cap \mathbf{L}_{b}$ with $\alpha>n / a$, Proposition 4.5 says that $\mathbf{\Lambda}_{a, p}^{\alpha} \cap \mathbf{L}_{b} \subset \mathbf{\Lambda}_{c, 1}^{n / c}$ where $c=\max (a, b)$, and, by Proposition $1.2(\mathrm{i}), \mathbf{\Lambda}_{c, 1}^{n / c} \subset \mathbf{C}_{0}$. Thus each of the spaces considered is contained in $\mathbf{C}_{0}$, and hence they are algebras by Lemma 1.5. It is clear that $D$ is dense in each of the spaces. We therefore have to do with an algebra $\mathbf{\Lambda}$ such that $\mathfrak{D} \subset \mathbf{\Lambda} \subset \mathbf{C}_{0}$, the inclusion being continuous, dense injection. The maximal ideal space of such an algebra is necessarily $R^{n}$, and so $\mathbf{\Lambda}$ is a regular self-adjoint, Banach algebra of functions on $R^{n}$. Again, since $D$ is dense in $\mathbf{\Lambda}$, in order to prove that $u^{t} f \rightarrow f$ in norm as $t \rightarrow 0$ for each $f \varepsilon \boldsymbol{\Lambda}$ it is sufficient to show that $u^{t}$ is bounded in the operator 
norm uniformly in $t<1$ and $u^{t} f \rightarrow f$ for each $f \varepsilon$ D. The operator norm of $u^{t}$ is majorized by $\Lambda_{a, p}^{\alpha}\left(u^{t}\right)+\left\|u^{t}\right\|_{\infty}$. Hence, for $\alpha \geqq n / a, \Lambda_{a, p}^{\alpha}\left(u^{t}\right)$ is dominated by $\Lambda_{a, p}^{\alpha}(u)$ when $t<1$ according to Lemma 1.2. When $\alpha<n / a$ we use Proposition 1.5 which shows that the operator norm is dominated by $\Lambda_{a, 1}^{n / a}\left(u^{t}\right)=\Lambda_{a, 1}^{n / a}(u)$.

We shall not give any detailed treatment of Lipschitz algebras here. In [3] we prove that there is a one-to-one correspondence between closed ideals of $\mathbf{\Lambda}_{n / \alpha, 1}^{\alpha}$ and closed sets in $R^{n}$ when $0<\alpha \leqq 1$. Analysis of the general ideal theory requires further machinery, but the "strong Ditkin condition" for maximal ideals in Lipschitz algebras with index of smoothness $\leqq 0$ can be deduced from elementary considerations.

Lemma 1.6. Suppose $f \varepsilon \mathbf{\Lambda}_{a, p}^{\alpha} \cap \mathbf{C}_{0}$ where $0<\alpha \leqq n / a$ and $u \varepsilon$ D. If $f(0)=0$ then $u^{t} f \rightarrow 0$ in norm as $t \rightarrow \infty$.

Proof. There is no loss in generality in assuming that $\|u\|_{\infty} \leqq 1$ and that $u(x)=0$ for $|x| \geqq 1$. Define $\delta(\epsilon)=\sup _{|x| \leqq \epsilon}|f(x)|$. Then $\left\|u^{t} f\right\|_{\infty} \leqq \delta\left(t^{-1}\right) \rightarrow 0$ as $t \rightarrow \infty$. Choose $k>\alpha$ and put $\omega(h ; t)=\left\|\Delta^{2 k}(h) u^{t} f\right\|_{a}$. It remains to prove that

$$
\lim _{t \rightarrow 0}\left\{\int\left[|h|^{-\alpha} \omega(h ; t)\right]^{p} d h\right\}^{1 / p}=0 .
$$

To do this we must estimate $\omega(h ; t)$. Since $u^{t}(x)=0$ for $|x|>t^{-1}$, we have $\left\|u^{t} f\right\|_{a} \leqq\left(b_{n} t^{n}\right)^{1 / a} \delta\left(t^{-1}\right)$. Therefore $\omega(h ; t) \leqq 4^{k}\left\|u^{t} f\right\|_{a} \leqq 4^{k} b_{n}^{1 / a} t^{n / a} \delta\left(t^{-1}\right)$ for all $t$ and $h$. As in the proof of Lemma 1.5 we may write

$$
\begin{aligned}
\Delta^{2 k}(h) f u^{t} & =\sum_{i=0}^{k-1}\left(\begin{array}{c}
2 k \\
i
\end{array}\right) \Delta^{i}(h) f \cdot \Delta^{2 k-i}(h) T^{i}(h) u^{t}+\sum_{i=k}^{2 k}\left(\begin{array}{c}
2 k \\
i
\end{array}\right) \Delta^{i}(h) f \cdot \Delta^{2 k-i}(h) T^{i}(h) u^{t} \\
& =\sigma_{1}(h ; t)+\sigma_{2}(h ; t) .
\end{aligned}
$$

For $\sigma_{2}$ we use the crude estimate

$$
\left\|\sigma_{2}(h ; t)\right\|_{a} \leqq 8^{k}\left\|\Delta^{k}(h) f\right\|_{a} .
$$

To majorize $\sigma_{1}$ we make two observations. First, the $k$ th derivative of $u$ is uniformly bounded; so $\left\|\triangle^{k}(h) u^{t}\right\|_{\infty} \leqq$ const. $|t h|^{k}$. Second, if $|h| \leqq(4 k t)^{-1}$ then $\sigma_{1}(h ; t ; x)=0$ when $|x|>3 / 2 t$. Thus, when $|h| \leqq(4 k t)^{-1}$, each summand in $\sigma_{1}(h ; t)$ is bounded by a constant multiple of $|t h|^{k} \delta\left(2 t^{-1}\right)$ while the expression vanishes outside of the ball of radius $3 / 2 t$. Hence there is a constant $c$ such that

$$
\left\|\sigma_{1}(h ; t)\right\|_{a} \leqq c|h|^{k} t^{k-n / a} \delta\left(2 t^{-1}\right) \quad \text { when }|h| \leqq(4 k t)^{-1} .
$$

The estimates

$$
\begin{array}{lll}
|h|^{-\alpha} \omega(h ; t) \leqq 4^{k} b_{n}^{1 / a}|h|^{-\alpha} t^{n / a} \delta\left(t^{-1}\right) & \text { when } & |h| \geqq(4 k t)^{-1} \\
|h|^{-\alpha} \omega(h ; t) \leqq 8^{k}|h|^{-\alpha}|| \Delta^{k}(h) f \|_{a}+c|h|^{k-\alpha} t^{k-n / a} \delta\left(2 t^{-1}\right) & \text { when } & |h| \leqq(4 k t)^{-1} \\
\text { give the required conclusion. } & &
\end{array}
$$


Proposition 1.7. Given $x_{0} \varepsilon R^{n}$ there is a sequence $\left\{u_{i}\right\} \subset D$ of functions each of which vanishes on a neighborhood of $x_{0}$ such that for each $f \boldsymbol{\varepsilon} \mathbf{\Lambda}_{a, p}^{\alpha} \cap \mathbf{C}_{0}$, where $0<\alpha \leqq n / a$, with $f\left(x_{0}\right)=0$ we have $\lim _{i \rightarrow \infty} u_{j} f=f$ in the norm of $\mathbf{\Lambda}_{a, p}^{\alpha} \cap$ $\mathbf{C}_{0}$.

Proof. We may assume $x_{0}=0$. Take $u \varepsilon D$ such that $u$ is identically 1 on a neighborhood of 0 , and put $u_{i}=u^{1 / i}-u^{i}$. For any $f \varepsilon \mathbf{\Lambda}_{a, p}^{\alpha} \cap \mathbf{C}_{0}$ we have $\lim _{i \rightarrow \infty} u^{1 / i} f=f$. By Lemma 1.6, $\lim _{j \rightarrow \infty} u^{i} f=0$ if $f(0)=0$.

In Theorem 5 we shall prove an inclusion relation between Lipschitz spaces and Lorentz spaces, namely, $\mathbf{\Lambda}_{a, p}^{\alpha} \subset \mathrm{L}_{b, p}$ where $1 / a-\alpha / n=1 / b$ provided that $\alpha>0$. The next is a partial converse which provides a useful and simple criterion.

Proposition 1.8. Suppose $f(x)$ is a decreasing function of $|x|$. If $f \in \mathbf{L}_{b, p}$ then also f $\varepsilon \mathbf{\Lambda}_{a, p}^{\alpha}$, where $1 / \alpha-\alpha / n=1 / b$, provided that $\alpha<1 / a$, i.e. $b<n a /(n-1)$.

Proof. We have $f(x)=f^{*}[v(x)]$ where $v(x)=b_{n}|x|^{n}$ and $f^{*}$ is the decreasing function on $(0, \infty)$ which is equi-measurable with $f$. Put $w=v(h)$, then

$$
\|\Delta(h) f\|_{a} \leqq \sum_{j=0}^{\infty} S_{j}(w)
$$

where we put $W_{i}=\{x: 2 j|h| \leqq x<2(j+1)|h|\}$ and

$$
S_{j}(w)=\left\{\int_{W_{j}}|f(x)-f(x+h)|^{a} d x\right\}^{1 / a} .
$$

The proposition will be proved when we show that $w^{-\alpha / n} \sum_{j=0}^{\infty} S_{j}(w) \varepsilon \mathcal{L}_{p}$, and this is a consequence of two assertions:

$$
w^{-\alpha / n} S_{0}(w) \varepsilon \mathscr{L}_{p}, \quad \text { and } w^{-\alpha / n} T(w) \varepsilon \mathfrak{L}_{p} \quad \text { where } \quad T(w)=\sum_{j=1}^{\infty} S_{j}(w) .
$$

We have

$$
\begin{aligned}
S_{0}(w) & \leqq\left\{\int_{W_{0}}|f(x)|^{a} d x\right\}^{1 / a}+\left\{\int_{W_{0}}|f(x+h)|^{a} d x\right\}^{1 / a} \\
& \leqq 2\left\{\int_{0}^{2^{n} w} f^{*^{a}}(v) d v\right\}^{1 / a} \leqq 2^{1+n / a}\left\{\int_{0}^{w} f^{*^{a}}(v) d v\right\}^{1 / a} \\
& \leqq 2^{1+n / a} \int_{0}^{w} f^{*}(v) v^{1 / a} d v
\end{aligned}
$$

since for any decreasing function $\varphi$ there is the estimate $\|\varphi\|_{a} \leqq \int \varphi(v) v^{1 / a} d v$. It follows that

$$
w^{-\alpha / n} S_{0}(w) \leqq 2^{1+n / a} \int_{0}^{w} f^{*}(v) v^{1 / b} k\left(w v^{-1}\right) d v
$$

where $k(s)=s^{-\alpha / n}$ for $s \geqq 1, k(s)=0$ for $s<1$. The right-hand side is a con- 
volution on the mutiplicative group $(0, \infty)$ of $k \varepsilon \mathfrak{L}_{1}$ with $f^{*} v^{1 / b} \varepsilon \mathfrak{L}_{p}$, and we get

$$
\left\|w^{-\alpha / n} S_{0}\right\|_{p} \leqq 2^{1+n / a}\|k\|_{1}\left\|f^{*} v^{1 / b}\right\|_{p}=2^{1+n / a}(n / \alpha)\|f\|_{b, p} .
$$

The treatment of $T(w)$ goes this way.

$$
\begin{aligned}
S_{j}(w) & \leqq \sup _{x \varepsilon W_{i}}|f(x)-f(x+h)| \cdot\left|W_{i}\right|^{1 / a} \\
& \leqq\left\{f^{*}\left[(2 j-1)^{n} w\right]-f^{*}\left[(2 j+3)^{n} w\right]\right\} \cdot 2^{2 n / a} j^{(n-1) / a} w^{1 / a} .
\end{aligned}
$$

Summation by parts gives

and so

$$
T(w) \leqq \text { const. } w^{1 / a} \sum_{i, \text { odd }} f^{*}\left(j^{n} w\right) j^{(n-1) / a-1} .
$$

$$
w^{-\alpha / n} T(w) \leqq \text { const. } \sum_{i, \text { odd }}\left(j^{n} w\right)^{1 / b} f^{*}\left(j^{n} w\right) j^{-e-1}
$$

where $e=n / b-(n-1) / a$. As long as $e>0$ we can use the Minkowski equality to obtain

$$
\begin{aligned}
\left\|w^{-\alpha / n} T(w)\right\|_{\nu} & \leqq \text { const. } \sum_{j, \text { odd }}\left\|\left(j^{n} w\right)^{1 / b} f^{*}\left(j^{n} w\right)\right\|_{p} j^{-e-1} \\
& =\text { const. }\|f\|_{b, p} \sum_{j, \text { odd }} j^{-e-1} \leqq \text { const. } e^{-1}\|f\|_{b, p} .
\end{aligned}
$$

The condition $e>0$ is equivalent to the condition $b<n a /(n-1)$.

Counterexample. If $f(x)=1$ for $|x| \leqq 1$ and $f(x)=0$ for $|x|>1$ then $f \varepsilon L_{b, p}$ for all $b$ and $p$ but $f \notin \mathbf{\Lambda}_{a, p}^{1 / a}$ for any $a$ with $1 \leqq a<\infty$.

Remark. In the limiting case $\alpha=1 / a$ the proof gives $\Lambda_{a, \infty}^{\alpha}(f)<\infty$, but not $f \varepsilon \mathbf{\Lambda}_{a, \infty}^{\alpha}$, since $\|\Delta(h) f\|_{a}=O\left(|h|^{\alpha}\right)$ but, in general, $O$ cannot be replaced by $o$.

In Proposition 1.8 the proof requires no appeal to Theorem 0 because the functions in question can be approximated by elements of $D$ throughout. A rather obvious method of approximating by elements of $D$ occur in the following.

Lemma 1.7. Suppose $\alpha>n / a$. If $f \varepsilon D C_{0}$ and $\Lambda_{a, p}^{\alpha}(f)<\infty$ (also $\left\|\Delta^{k}(h) f\right\|_{a}=$ $o\left(|h|^{\alpha}\right)$ for some $k>\alpha$ if $\left.a=\infty\right)$ then $f \varepsilon \mathbf{\Lambda}_{a, p}^{\alpha}$.

Proof. Take $u \varepsilon \mathbb{D}$ with $u=1$ on a neighborhood of the origin. Then $u^{t} f \varepsilon \mathbb{D}$ for all $t>0$, and it suffices to show that $\lim _{t \rightarrow 0} \Lambda_{a, p}^{\alpha}\left\{\left(1-u^{t}\right) f\right\}=0$. For this we go back to the proof of Lemma 1.5. For $k>\alpha$ the Leibniz formula would give

$$
\begin{aligned}
\| \Delta^{2 k}(h)(1 & \left.-u^{t}\right) f \|_{a} \\
\leqq & \sum_{i=k}^{2 k}\left(\begin{array}{c}
2 k \\
i
\end{array}\right)\left\|\Delta^{2 k-i}(h) T^{i}(h)\left(1-u^{t}\right) \cdot \Delta^{i}(h) f\right\|_{a}+c\|f\|_{\infty}\left\|\Delta^{k}(h) u^{t}\right\|_{a} .
\end{aligned}
$$

For fixed $h$, each term in the summation is majorized by a constant multiple of $\left\|\Delta^{k}(h) f\right\|_{a}$ and tends to 0 as $t \rightarrow 0$. Using dominated convergence, we have

$$
\Lambda_{a, p}^{\alpha}\left\{\left(1-u^{t}\right) f\right\} \leqq o(1)+c\|f\|_{\infty} \Lambda_{a, p}^{\alpha}\left(u^{t}\right) \text { on } t \rightarrow 0 .
$$


By Lemma 1.2, $\Lambda_{a, p}^{\alpha}\left(u^{t}\right)=t^{\alpha-n / a} \Lambda_{a, p}^{\alpha}(u)$; this tends to 0 as $t \rightarrow 0$ when $\alpha>n / a$.

Remark. With more care the same proof works for $\alpha=n / a>0$.

By the Corollary to Proposition 1.5, $\lim _{t \rightarrow 0} \Lambda_{a, p}^{\alpha}\left\{\left(1-u^{t}\right) f\right\}=0$ when $f \varepsilon \mathbf{\Lambda}_{a, p}^{\alpha}$ and $0<\alpha<n / a$. This cannot, however, be used to prove Theorem 0 ; so a trick is employed.

Lemma 1.8. If $f \varepsilon \mathscr{D} C_{0}$ and $\Lambda_{a, p}^{\alpha}(f)<\infty$ (also $\left\|\Delta^{k}(h) f\right\|_{a}=o\left(|h|^{\alpha}\right)$ for some $k>\alpha$ if $p=\infty$ ) then $f \varepsilon \boldsymbol{\Lambda}_{a, p}^{\alpha}$.

Proof. Take $w \varepsilon \subseteq$ such that the Fourier transform $\hat{w}$ is identically 1 on a neighborhood of the origin and vanishes outside a compact set. Then $\lim _{t \rightarrow 0} \Lambda_{a, p}^{\alpha}\left\{f-\left(w_{t}-w_{1 / t}\right) * f\right\}=0$. Hence it suffices to prove that $g=\left(w_{t}-\right.$ $\left.w_{1 / t}\right) * f \varepsilon \boldsymbol{\Lambda}_{a, p}^{\alpha}$ for each $t>0$. The hypotheses on $g$ are: $\Lambda_{a, p}^{\alpha}(g)<\infty, g \varepsilon \mathfrak{D} C_{0}$, and, for some $\epsilon>0$, the Fourier transform of the tempered distribution $g(x) d x$ has its support in $\left\{\xi \varepsilon R^{n} ; \epsilon<|\xi|<\epsilon^{-1}\right\}$. Let $j$ be an integer so large that $\alpha+$ $2 j>n / a$. Then there exists $r \varepsilon S$ whose Fourier transform is such that

$$
\hat{r}(\xi)=\left(-4 \pi^{2}|\xi|^{2}\right)^{-i} \text { when } \epsilon \leqq|\xi| \leqq \epsilon^{-1} .
$$

Let Lap signify the Laplacian. We have

$$
g=\operatorname{Lap}^{i}(r * g) \text {. }
$$

Since $r \varepsilon \mathcal{S}$ it follows that $r \varepsilon \Lambda_{1, \infty}^{2 j}$. Put $c=\Lambda_{1, \infty}^{2 j}(r)$. Then, as in the proof of Proposition 1.3, $\Lambda_{a, p}^{\alpha+2 i}(r * g) \leqq c \Lambda_{a, p}^{\alpha}(g)<\infty$. Lemma 1.7 tells us that $r * g \varepsilon$ $\Lambda_{a, p}^{\alpha+2 i}$. We now invoke Proposition 6.1 which, in this case $\left(\operatorname{Lap}^{i}=R^{-2 i}\right.$ ), asserts that $\operatorname{Lap}^{j} \operatorname{maps} \mathbf{\Lambda}_{a, p}^{\alpha+2 j}$ into $\boldsymbol{\Lambda}_{a, p}^{\alpha}$.

2. The spaces ${ }_{\mathrm{a}} L_{\mathrm{p}}$ and $K_{\mathrm{a}, \mathrm{p}}$. Let $\Phi$ be a cone of positive Baire functions on $R^{n}$ furnished with a norm \|\| such that

(a) if $\left\{\varphi_{j}\right\} \subset \Phi$ and $\sum\left\|\varphi_{j}\right\|<\infty$ then $\sum \varphi_{i} \varepsilon \Phi$

(b) if $\varphi_{1}, \varphi_{2} \varepsilon \Phi$ and $\psi(\xi)=\inf \left\{\varphi_{1}(\xi), \varphi_{2}(\xi)\right\}$ then $\psi \varepsilon \Phi$ and $\|\psi\| \leqq \min$ $\left(\left\|\varphi_{1}\right\|,\left\|\varphi_{2}\right\|\right)$.

For fixed $\varphi \varepsilon \Phi, 1 \leqq a \leqq \infty$, and $1 \leqq p \leqq \infty$ we define ${ }_{a} \mathbf{L}_{p}(\varphi)$ to be the Banach space of measurable functions with finite ${ }_{a}\|\|_{p}^{\varphi}$ norm where

$$
{ }_{a}\|g\|_{p}^{\varphi}=\left\{\int|g(\xi)|^{a} \varphi^{1-a / p}(\xi) d \xi\right\}^{1 / a}\|\varphi\|^{1 / p-1 / a} .
$$

When $a=\infty$ the interpretation is

$$
{ }_{\infty}\|g\|_{p}^{\varphi}=\|\varphi\|^{1 / p} \operatorname{ess} \sup _{\xi}|g(\xi)| \varphi^{-1 / p}(\xi) .
$$

It is also clear that ${ }_{p} \mathbf{L}_{p}(\varphi)=\mathbf{L}_{p}$ for all $\varphi$.

The spaces ${ }_{a} \mathbf{L}_{p}$ are defined this way:

if

$$
a \geqq p, \quad{ }_{a} \mathrm{~L}_{p}=\bigcup_{\varphi \varepsilon \Phi}{ }_{a} \mathbf{L}_{p}(\varphi),
$$


if

$$
a \leqq p, \quad{ }_{a} \mathbf{L}_{p}=\bigcap_{\varphi \varepsilon \Phi}{ }_{a} \mathbf{L}_{p}(\varphi) .
$$

When $1 \leqq a \leqq p$ the nature of the ${ }_{a} \mathbf{L}_{p}$ spaces is clear. Turning to the case $p \leqq a \leqq \infty$ we see that ${ }_{p} \mathrm{~L}_{p}=\mathrm{L}_{p}$ and ${ }_{\infty} \mathrm{L}_{p}$ has the norm

$$
{ }_{\infty}\|g\|_{p}=\inf \left\{\|\varphi\|^{1 / p}:|g|^{p} \leqq \varphi \varepsilon \Phi\right\} .
$$

The other ${ }_{a} \mathbf{L}_{p}$ spaces are natural intermediate spaces in the sense of Calderón [2; §13].

Proposition 2.1. Suppose $1 \leqq p \leqq \infty, 1 \leqq a \leqq \infty$.

(i) Each ${ }_{a} \mathbf{L}_{p}$ is a Banach space, and if $|f| \leqq|g|$ where $g \varepsilon_{a} \mathbf{L}_{p}$ then $f \varepsilon_{a} \mathbf{L}_{p}$.

(ii) If $0<\sigma=a / p<1$ then ${ }_{a} \mathbf{L}_{p}$ is the complex interpolation space: ${ }_{a} \mathbf{L}_{p}=$ $\left.\left(\mathbf{L}_{p}\right)^{\sigma}{ }_{\infty} \mathrm{L}_{p}\right)^{1-\sigma}$.

(iii) If $\Phi \subset \mathrm{L}_{1}$ then ${ }_{a} \mathbf{L}_{p} \subset{ }_{b} \mathbf{L}_{p}$ whenever $a \geqq b$.

(iv) If convolution is a bicontinuous operation on $\Phi$ then each ${ }_{a} \mathrm{~L}_{1}$ is a Banach algebra under convolution.

Proof. To prove statement (iii) we use the Hölder inequality:

$$
\int\left|g \varphi^{-1 / p}\right|^{b} \varphi d \xi \leqq\left(\int\left|g \varphi^{-1 / p}\right|^{a} \varphi d \xi\right)^{b / a}\left(\int \varphi d \xi\right)^{1-b / a} .
$$

If $\int \varphi(\xi) d \xi \leqq\|\varphi\|$ then it follows that ${ }_{b}\|g\|_{p}^{\varphi} \leqq{ }_{a}\|g\|_{p}^{\varphi}$. This gives directly ${ }_{b}\|g\|_{b} \leqq{ }_{a}\|g\|_{p}$ whenever $b \leqq a \leqq p$ or $p \leqq b \leqq a$, but the same result holds when $b \leqq p \leqq a$ for then ${ }_{b}\|g\|_{p} \leqq\|g\|_{p} \leqq{ }_{a}\|g\|_{p}$. At the loss of introducing constants in the estimates, it suffices merely to assume that $\Phi \subset L_{1}$. Statement (i) is obvious if $a \leqq p$. When $a \geqq p$, assertion (i) and (iv) can be proved as is done for the case $p=1$ in Beurling [1]. These statements are, however, consequences of more general considerations and (ii). To see (ii), put $\mathbf{X}_{1}=\mathbf{L}_{p}$ and $\mathrm{X}_{0}={ }_{\infty} \mathrm{L}_{p}$. Recall that the norm in $\mathrm{X}_{0}$ is

$$
{ }_{\infty}\|g\|_{p}=\inf \left\{\|\varphi\|^{1 / p}:|g|^{p} \leqq \varphi \varepsilon \Phi\right\} .
$$

It is therefore clear that $\mathbf{X}_{0}$ is a Banach lattice in the sense of Calderón [2: $\S 13.1]$ and, of course, $\mathbf{X}_{1}$ is. The complex intermediate space $\mathbf{X}_{\sigma}=\mathbf{X}_{0}^{1-\sigma} \mathbf{X}_{1}^{\sigma}$ is, according to $[2 ; \S 13.5]$, the lattice of measurable functions of finite norms where the norm is defined by

$$
\|g\|=\inf \left\{\|h\|_{X_{1}}^{\sigma}:|g| \leqq|h|^{\sigma}|\psi|^{1-\sigma}, \quad|\psi|_{x_{0}} \leqq 1\right\} .
$$

In our case we may assume $|\psi|=\varphi^{1 / p}$ where $\varphi \varepsilon \Phi$ and $\|\varphi\|=1$. Therefore $|h|=|g|^{1 / \sigma} \varphi^{1 / p(1-1 / \sigma)} ;$ so

$$
\|g\|=\inf \|h\|_{p}^{\sigma}=\inf \left\{\int|g|^{p / \sigma} \varphi^{1-1 / \sigma} d \xi\right)^{\sigma / p}
$$

where the infimum is taken over $\varphi \varepsilon \Phi$ with $\|\varphi\|=1$. This is exactly the definition of the ${ }_{a} \mathbf{L}_{p}$-norm for $a=p / \sigma$. 
We shall not make any use of the interpolation properties of the ${ }_{a} \mathbf{L}_{p}$ spaces beyond what is needed for the very important duality relation.

Proposition 2.2. For $1 \leqq p<\infty, 1 \leqq a<\infty$, the dual space of ${ }_{a} \mathbf{L}_{p}$ is ${ }_{a^{\prime}} \mathbf{L}_{p^{\prime}}$.

Proof. Let $\mathrm{L}_{a}(\varphi)$ signify the Lebesgue space with respect to the measure $\varphi(\xi) d \xi$, it has the norm

$$
\|h\|_{a}^{\varphi}=\left\{\|\varphi\|^{-1} \int|h(\xi)|^{a} \varphi(\xi) d \xi\right\}^{1 / a} .
$$

For $1 \leqq a<\infty$, the dual space of $\mathbf{L}_{a}(\varphi)$ is $\mathbf{L}_{a^{\prime}}(\varphi)$. Now $g \varepsilon_{a} \mathbf{L}_{p}(\varphi)$ iff $g \varphi^{-1 / p} \varepsilon$ $\mathbf{L}_{a}(\varphi)$. Consequently the linear functionals $F$ on ${ }_{a} \mathbf{L}^{p}(\varphi)$ are precisely represented in the form

$$
F(g)=\int f(\xi) g(\xi) d \xi=\int\left(f \varphi^{-1 / p^{\prime}}\right)\left(g \varphi^{-1 / p}\right) \varphi d \xi,
$$

where $f \varepsilon_{a}, \mathbf{L}_{p^{\prime}}(\varphi)$ and $\|F\|={ }_{a^{\prime}}\|f\|_{p^{\prime}}^{\varphi}$. When $a \geqq p$ we have ${ }_{a} \mathbf{L}_{p}=\bigcup_{\varphi \in \Phi}{ }_{a} \mathbf{L}_{p}(\varphi)$, and it is obvious that

$$
\left({ }_{a} L_{p}\right)^{\prime}=\left(\cup{ }_{a} L_{p}(\varphi)\right)^{\prime}=\cap\left({ }_{a} L_{p}(\varphi)\right)^{\prime}=\bigcap_{a^{\prime}} L_{p^{\prime}}(\varphi)={ }_{a^{\prime}} \mathbf{L}_{p^{\prime}} .
$$

This proves the assertion when $1 \leqq p \leqq a<\infty$. Since the set-theoretic union is only in special cases the functorial union for Banach spaces, it seems difficult to dualize the argument to handle $a \leqq p$. However it follows from Proposition 2.1 (ii), [2; §12.2], and the reflexivity of $\mathbf{L}_{p}$ that ${ }_{a} \mathbf{L}_{p}$ is reflexive when $1<p \leqq$ $a<\infty$. This takes care of the remaining cases.

Henceforth we shall only consider the particular class $\Phi$ given below.

Definition. $\Phi$ consists of the functions $\varphi \varepsilon L_{1}$ such that $\varphi(\xi)$ is a decreasing function of $\xi$. The natural norm for $\Phi$ is $\|\varphi\|=\int \varphi(\xi) d \xi$, and $\Phi$ is stable under convolution.

Recall the notation $v(\xi)=b_{n}|\xi|^{n}$, the volume of the ball of radius $|\xi|$. The spaces ${ }_{a} \mathbf{L}_{p}$ have an interesting alternative description.

Proposition 2.3. Given a measurable function $g$ and a positive number $u$, put

$$
G(u)=\left\{u^{-1} \int_{v(\xi) \leq u}|g(\xi)|^{a} d \xi\right\}^{1 / a}
$$

(so $G(u)$ is the $L_{a}$-average of $|g|$ over the ball of volume $u$ centered at the origin), and put

$$
\widetilde{G}(u)=\left\{u \int_{v(\xi) \geqq u}|g(\xi)|^{a} v^{-2}(\xi) d \xi\right\}^{1 / a} .
$$

(i) If $a<p$ then $\|g\|=\left\{\int_{0}^{\infty} G^{p}(u) d u\right\}^{1 / p}$ is an equivalent norm on ${ }_{a} \mathrm{~L}_{p}$.

(ii) It is always true that $\|g\|=\left\{\int_{0}^{\infty} \widetilde{G}^{p}(u) d u\right\}^{1 / p}$ is an equivalent norm on ${ }_{a} \mathrm{~L}_{0}$.

Proposition 2.3 is merely a special case of Proposition 2.4 below, and we stated Proposition 2.3 only to give some concrete notion of the ${ }_{a} \mathbf{L}_{\nu}$ spaces. To be sure, 
Proposition 2.1 and 2.2 for the special choice of $\Phi$ are easy consequences of Proposition 2.3 (ii) which does not depend on the earlier results.

The ${ }_{a} \mathbf{L}_{p}$ spaces are not convenient to use directly for purposes of Fourier analysis. We therefore introduce another class of spaces.

Definition. The Banach spaces $\mathbf{K}_{a, p}$ are defined for $1 \leqq a \leqq \infty, 1 \leqq p \leqq \infty$ by $g \varepsilon \mathbf{K}_{a, p}$ iff $g v^{1 / a-1 / p} \varepsilon_{a} \mathbf{L}_{p}$.

This definition of the $\mathbf{K}_{a, p}$ spaces may seem rather awkward. The main theorem of this section (Proposition 2.4) furnishes a very general alternative definition which is vital for later use.

Definition. A $K$-kernel is a non-trivial function $\hat{\kappa}$ defined on $R^{n} \times R^{n}$ with the properties

(a) $|\hat{\kappa}(T \xi ; h)|=\left|\hat{\kappa}\left(\xi ; T^{\prime} h\right)\right|$ for any linear transformation $T$ which is a dilation (scalar multiple) of an orthogonal transformation, $T^{\prime}$ being the transposed;

(b) for some $\theta \neq 0$ and some $\epsilon \neq 0$,

$$
\hat{\kappa}(\theta ; h)\left\{|h|^{\epsilon}+|h|^{-\epsilon}\right\} \text { is bounded. }
$$

Proposition 2.4. Ket $\hat{\kappa}$ be a $K$-kernel. For a measurable function $g$ define $K_{a, v}(g)$ to be the $\mathfrak{L}_{p}$-norm of $\omega$ where $\omega(h)$ is the $\mathbf{L}_{a}$-norm of $g \hat{\kappa}(\cdot ; h)$. Then $K_{a, p}$ is an equivalent norm on $\mathbf{K}_{a, p}$.

Written out in full the $K_{a, p}$ norm is

$$
K_{a, p}(g)=\left\{\int\left[\int|g(\xi)|^{a}|\hat{k}(\xi ; h)|^{a} d \xi\right]^{p / a} d h\right\}^{1 / p} .
$$

Our proof of Proposition 2.4 is quite complicated. We demonstrate four lemmas.

Lemma 2.1. If $p \leqq a$ then ${ }_{a}\left\|g v^{1 / a-1 / p}\right\|_{p} \leqq$ const. $K_{a, p}(g)$.

Lemma 2.2. If $p \geqq a$ then ${ }_{a}\left\|g v^{1 / a-1 / p}\right\|_{p} \leqq$ const. $K_{a, p}(g)$.

Lemma 2.3. If $p \leqq a$ then $K_{a, p}(g) \leqq$ const. ${ }_{a}\left\|g v^{1 / a-1 / p}\right\|_{p}$.

Lemma 2.4. If $p \geqq a$ then $K_{a, p}(g) \leqq$ const. $a\left\|g v^{1 / a-1 / p}\right\|_{p}$.

Lemmas 2.1 and 2.2 do not make use of property (b) of $K$-kernels, but none of the lemmas is banal. Proposition 2.3 is technically a little simpler to prove than the general Proposition 2.4, but the ideas are essentially the same, and we prefer to exhibit Proposition 2.3 as a corollary of Proposition 2.4. The proofs of the four lemmas above is deferred until Section $2^{*}$.

Proof of Proposition 2.3( $(i)$. Let $a$ and $p$ be given with $\epsilon=1 / a-1 / p>0$. Choose the $K$-kernel to be

$$
\hat{\kappa}(\xi ; h)=v^{\epsilon}(\xi) v^{\epsilon}(h) \text { if } \quad v(\xi) v(h) \leqq 1,=0 \text { otherwise. }
$$

According to Proposition 2.4 the ${ }_{a}\|g\|_{p}$ norm is equivalent to $K_{a, p}\left(g v^{-6}\right)$. On writing $v(h)=u^{-1}$ we have for the $\omega$ corresponding to $g v^{-\epsilon}$, 


$$
\omega(h)=u^{-\epsilon}\left\{\int_{v(\xi) \leqq u}|g(\xi)|^{a} d \xi\right\}^{1 / a}=u^{1 / p} G(u) .
$$

Thus the $\mathscr{L}_{p}$-norm of $\omega$ is the $\mathrm{L}_{p}$-norm of $G$.

Proof of Proposition 2.3(ii). The case $a=\infty=p$ is trivial; so we may suppose that $\delta=1 / a+1 / p>0$. Choose the $K$-kernel to be

$$
\hat{\kappa}(\xi ; h)=v^{-\delta}(\xi) v^{-\delta}(h) \quad \text { if } \quad v(\xi) v(h) \geqq 1,=0 \quad \text { otherwise. }
$$

At the expense of introducing yet another index we make the

Definition. The Banach space $\mathrm{K}_{a, p}^{\alpha}$ with $\alpha$ real consists of the measurable functions $g$ such that $g v^{\alpha / n} \varepsilon \mathrm{K}_{a, p}$.

It is worthwhile noting that if the $\mathbf{K}_{a, p}$ norm is given by

$$
K_{a, p}(g)=\left\{\int \omega^{p}(h) d h\right\}^{1 / p}
$$

where $\omega(h)=\| g \hat{\kappa}\left(\cdot ; h \|_{a}\right.$, then

$$
K_{a, p}^{\alpha}(g)=\left\{\int\left[|h|^{-\alpha} \omega(h)\right]^{p} d h\right\}^{1 / p} .
$$

There is a simple basic inclusion relation among the $\mathbf{K}_{a, p}^{\alpha}$ spaces.

Proposition 2.5. Suppose $\beta \leqq \alpha, 1 / a+\alpha / n=1 / b+\beta / n$, and $p \leqq q$. Then $\mathbf{K}_{a, p}^{\alpha} \subset \mathbf{K}_{b, q}^{\beta}$.

Proof. To say that $g \varepsilon \mathrm{K}_{a, p}^{\alpha}$ is to say that $g v^{e} \varepsilon_{a} \mathrm{~L}_{p}$ where $e=\alpha / n+1 / a-$ $1 / p$. Under the hypotheses, $a \geqq b$; so by Proposition 2.1 (iii) we have $g v^{\circ} \varepsilon_{b} \mathbf{L}_{p}$; but this says $g \varepsilon \mathbf{K}_{b, p}^{\beta}$. Observe that for $\varphi \varepsilon \Phi,|\varphi(\xi) v(\xi)| \leqq\|\varphi\|$; hence

whenever $p \leqq q$.

$$
\begin{aligned}
{ }_{b}\left\|g v^{\beta / n}\right\|_{a} & =\left\{\int\left|g v^{\beta / n}\right|^{b}(\varphi v)^{1-b / a} d \xi\right\}^{1 / b}\|\varphi\|^{1 / a-1 / b} \\
& \leqq\left\{\int\left|g v^{\beta / n}\right|^{b}(\varphi v)^{1-b / p} d \xi\|\varphi\|^{b / p-b / a}\right\}^{1 / b}\|\varphi\|^{1 / a-1 / b} \\
& ={ }_{b}\left\|g v^{\beta / n}\right\|_{p}
\end{aligned}
$$

The spaces ${ }_{a} \mathbf{L}_{p}=\mathbf{K}_{a, p}^{n(1 / p-1 / a)}$ are, for fixed $p$ and variable $a$, a decreasing family of spaces containing $\mathbf{L}_{p}={ }_{p} \mathbf{L}_{p}$. For fixed $a$ and variable $p$ the spaces $\mathbf{K}_{a, p}$ form an increasing family containing $\mathbf{L}_{a}=\mathbf{K}_{a, a}$. Neither of these families has any relation to the Lorentz spaces $\mathbf{L}_{a, p}$ beyond the immediate consequences of ${ }_{p} \mathbf{L}_{p}=\mathbf{L}_{p}$ and $\mathbf{K}_{a, a}=\mathbf{L}_{a}$. For many applications, however, the following is useful.

Proposition 2.6. If $\alpha>0$ and $1 / a+\alpha / n=1 / b$ then $\mathbf{K}_{a, p}^{\alpha} \subset \mathbf{L}_{b, p}$.

Proof. Put $\epsilon=\alpha / n$. Choose the $K$-kernel $\hat{\kappa}$ to be

$$
\hat{\kappa}(\xi ; h)=v^{-\epsilon}(\xi) v^{-\epsilon}(h) \quad \text { if } \quad v(\xi) v(h) \geqq 1,=0 \quad \text { otherwise. }
$$


Then the $\mathbf{K}_{a, p}^{\alpha}$-norm of $g$ is equivalent to the $\mathfrak{L}_{p}$-norm of $w$ where

$$
w^{a}(u)=u^{\epsilon a} \int_{v(\xi) \geqq u}|g(\xi)|^{a} d \xi .
$$

Taking the decreasing rearrangement we have

$$
\begin{aligned}
w^{a}(u) & \geqq u^{\epsilon a} \int_{u}^{\infty} g^{*}(v) d v \geqq u^{\epsilon a} \int_{u}^{2 u} g^{* a}(v) d v \\
& \geqq u^{\epsilon a+1} g^{*^{a}}(2 u) .
\end{aligned}
$$

Since $\epsilon+1 / a=1 / b$ this gives $w(u) \geqq u^{1 / b} g^{*}(2 u)$; therefore $w \varepsilon \mathcal{L}_{p}$ implies $g \varepsilon \mathrm{L}_{b, p}$.

It is worthwhile rewriting Proposition 2.2 for the $\mathrm{K}_{a, p}^{\alpha}$ spaces.

Proposition 2.7. For all real $\alpha, 1 \leqq p<\infty$, and $1 \leqq a<\infty$, the dual space of $\mathbf{K}_{a, p}^{\alpha}$ is $\mathbf{K}_{a^{\prime}, w^{\prime}}^{-\alpha}$.

A set $E \subset R^{n}$ will be called lacunary if

$$
\int_{E} \varphi(\xi) v(\xi) d \xi<\infty \quad \text { for all } \varphi \varepsilon \Phi .
$$

For example, if $\lambda>1$, the set

$$
E=\bigcup_{k=0}^{\infty}\left\{\xi: \lambda^{k}-1<v(\xi) \leqq \lambda^{k}\right\}
$$

is lacunary. A function $g$ is lacunary if

$$
\{\xi: g(\xi) \neq 0\} \text { is lacunary. }
$$

Most of the familiar properties of lacunary functions in harmonic analysis will be derived from a simple observation.

Proposition 2.8. If $g$ is lacunary and $g \in \mathbf{K}_{a, p}$ then $g \varepsilon \mathbf{K}_{b, p}$ for all $b$ with $1 \leqq b \leqq a$.

Proof. If $\varphi \varepsilon \Phi, b \leqq a$, and $E$ is a lacunary set then by the Hölder inequality

$$
\left\{\int_{E}|g|^{b}(\varphi v)^{-b / \nu} \varphi v d \xi\right\}^{1 / b} \leqq\left\{\int_{E}|g|^{a}(\varphi v)^{-a / p} \varphi v d \xi\right\}^{1 / a}\left\{\int_{E} \varphi v d \xi\right\}^{1 / b-1 / a} .
$$

From the Principle of Uniform Boundedness it follows that for each lacunary set $E$ there is a constant $M_{E}$ such that

$$
\int_{E} \varphi(\xi) v(\xi) d \xi \leqq M_{E}\|\varphi\| \text {. }
$$

Hence, if we put $K_{a, p}(g)={ }_{a}\left\|g v^{1 / a-1 / p}\right\|_{p}$, we have

$$
K_{b, p}(g) \leqq M_{E}^{1 / b-1 / a} K_{a, p}(g)
$$

for all functions $g$ which vanish outside $E$. 
2*. Proof of Proposition 2.4. For a measurable function $g$ put

$$
N_{a, p}(g)=\inf \sup _{\varphi}\left\{\int|g(\xi)|^{a}[\varphi(\xi) v(\xi)]^{1-a / p} d \xi\right\}^{1 / a}\|\varphi\|^{1 / p-1 / a},
$$

where inf or sup is chosen according to whether $a \geqq p$ or $a \leqq p$. The norm $N_{a, p}$ may be taken as the standard norm for the space $\mathbf{K}_{a, p}$.

Given a kernel $\hat{\kappa}$ satisfying the condition

(a) $|\hat{\kappa}(T \xi ; h)|=\left|\hat{\kappa}\left(\xi ; T^{\prime} h\right)\right|$ for any $T$ which is a dilation of an orthogonal transformation, we put

where

$$
K_{a, p}(g)=\left\{\int \omega^{p}(h) d h\right\}^{1 / p}, \quad d h=v^{-1}(h) d h
$$

$$
\omega(h)=\left\{\int|g(\xi)|^{a}|\hat{\kappa}(\xi ; h)|^{a} d \xi\right\}^{1 / a} .
$$

Proposition 2.4 has two parts. The first part says that if $\hat{\kappa}$ is non-trivial then the norm $K_{a, p}$ dominates the norm $N_{a, p}$. This is the content of Lemmas 2.1 and 2.2. The second part of the proposition asserts that under additional hypotheses on the kernel $\hat{\kappa}$ the norm $N_{a, p}$ dominates the norm $K_{a, p}$.

To simplify the notation we shall often write $\sigma=p / a$ and $1 / \tau=1-1 / \sigma$.

When $a \geqq p$ we define

A precise statement is

$$
k_{a, p}=\sup _{\xi}\left\{\int_{v(h) \leqq 1}|\hat{\kappa}(\xi ; h)|^{p} v^{1-p / a}(h) d h\right\}^{1 / \nu}
$$

Lemma 2.1. $N_{a, p} \leqq k_{a, p}^{-1} K_{a, p}$ when $1 \leqq p \leqq a \leqq \infty$.

Proof. Since the $K_{a, p}$ norm formed with the kernel $\hat{\kappa}(r \xi ; h)$ is the same as that formed with the kernel $\hat{\kappa}(\xi, h)$, it suffices to prove that $N_{a, p} \leqq k^{-1} K_{a, p}$ where

$$
k^{p}=\int_{v(h) \leqq 1}|\hat{\kappa}(\theta ; h)|^{p} v^{1-p / a}(h) d h, \quad v(\theta)=1 .
$$

The invariance properties of $\kappa$ give further

$$
\begin{aligned}
k^{p} & =\int_{v(\xi) v(h) \leqq 1}|\hat{\kappa}(\xi ; h)|^{p}[v(\xi) v(h)]^{1-\sigma} d h \\
& =\int_{v(\xi) v(h) \leqq 1}\left\{|\hat{\kappa}(\xi ; h)|^{a} \omega^{a-p}(h)\right\}^{\sigma}\left\{\omega^{p}(h) v(\xi) v(h)\right\}^{1-\sigma} d h,
\end{aligned}
$$

whatever the function $\omega \geqq 0$. Define functions $\varphi$ and $\psi$ by

$$
\begin{aligned}
\varphi(\xi) & =\int_{v(\xi) v(h) \leqq 1} \omega^{p}(h) d h, \\
\psi^{a}(\xi) & =\int|\hat{\kappa}(\xi ; h)|^{a} \omega^{a-p}(h) d h .
\end{aligned}
$$


The Hölder inequality yields

$$
k^{p} \leqq \psi^{p}(\xi)[\varphi(\xi) v(\xi)]^{1-\sigma} .
$$

The function $\varphi$ clearly has the property that $\varphi(\xi)$ is a decreasing function of $|\xi|$. Moreover

$$
\|\varphi\|=\int \varphi(\xi) d \xi=\int \omega^{p}(h) d h .
$$

Now, given a measurable function $g$, put

$$
\omega^{a}(h)=\int|g(\xi)|^{a}|\hat{\kappa}(\xi ; h)|^{a} d \xi .
$$

Multiplying by $\omega^{p-a}(h)$ and integrating $d h$, we get

$$
\int \omega^{p}(h) d h=\int|g(\xi)|^{a} \psi^{a}(\xi) d \xi .
$$

We have computed that $[\varphi(\xi) v(\xi)]^{1-a / p} \leqq k^{-a} \psi^{a}(\xi)$. Therefore

$$
\int|g(\xi)|^{a}[\varphi(\xi) v(\xi)]^{1-a / p} d \xi \leqq k^{-a} \int \omega^{p}(h) d h=k^{-a}\|\varphi\| .
$$

It is immediate that $N_{a, p}(g) \leqq k^{-1}\|\varphi\|^{1 / p}=k^{-1} K_{a, p}(g)$.

When $a \leqq p$ we define

$$
c_{a, p}=\sup _{\xi}\left\{\int_{v(h) \geqq 1}|\hat{\kappa}(\xi ; h)|^{a} v^{a / p-1}(h) d h\right\}^{1 / a} .
$$

Another precise statement is

Lemma 2.2. $N_{a, p} \leqq c_{a, p}^{-1} K_{a, p}$ when $1 \leqq p \leqq a \leqq \infty$.

Proof. As in the proof of Lemma 2.1 it suffices to prove that $N_{a, p} \leqq c^{-1} K_{a, p}$ where

$$
c^{a}=\int_{v(\xi) v(h) \geqq 1}|\hat{\kappa}(\xi ; h)|^{a}[v(\xi) v(h)]^{-1 / \tau} d h .
$$

Choose $\varphi \varepsilon \Phi$, and put $f(v)=\varphi^{*}(v) v$. Since $\varphi^{*}$ is decreasing, we have

It follows that

$$
\varphi(\xi) v(\xi) \leqq f\left(u^{-1}\right) v(\xi) u \quad \text { whenever } v(\xi) u \geqq 1 .
$$

$$
[\varphi(\xi) v(\xi)]^{1 / \tau} \leqq c^{-a} \int_{v(\xi) v(h) \geqq 1}|\hat{\kappa}(\xi ; h)|^{a} f^{1 / \tau}\left[v^{-1}(h)\right] d h .
$$

Given a measurable function $g$, multiplying the above equation by $|g(\xi)|^{a}$ and integrating $d \xi$, we get

$$
\begin{aligned}
\int|g(\xi)|^{a}[\varphi(\xi) v(\xi)]^{1 / \tau} d \xi & \leqq c^{-a} \int \omega^{a}(h) f^{1 / \tau}\left[v^{-1}(h)\right] d h \\
& \leqq c^{-a}\left\{\int \omega^{p}(h) d h\right\}^{\sigma}\left\{\int f\left[v^{-1}(h)\right] d h\right\}^{\tau}
\end{aligned}
$$


from the Hölder inequality, where

$$
\omega^{a}(h)=\int|g(\xi)|^{a}|\hat{\kappa}(\xi ; h)|^{a} d \xi .
$$

Since $\int f\left[v^{-1}(h)\right] d h=\int f(u) d u=\|\varphi\|$, the required inequality follows immediately.

For efficient estimates in Lemma 2.3 we put

$$
\begin{gathered}
k_{p}=\left\{\int|\hat{\kappa}(\xi ; h)|^{p} d h\right\}^{1 / p}, \\
\tilde{k}_{p}=\sup _{\varphi}\left\{\int \sup _{\xi}|\hat{\kappa}(\xi ; h)|^{p} \varphi(\xi) v(\xi) d h\right\}^{1 / p}\|\varphi\|^{-1 / p} .
\end{gathered}
$$

The desired result is

Lemma 2.3. $K_{a, p} \leqq k_{p}^{p / a} \tilde{k}_{p}^{1-p / a} N_{a, p}$ when $1 \leqq p \leqq a \leqq \infty$, and if $\hat{\kappa}$ is $a$ $K$-kernel then both $k_{p}$ and $\tilde{k}_{p}$ are finite.

Proof. Let $\varphi \varepsilon \Phi$ be given and define $\lambda$ by

$$
\lambda^{p}(h)=\sup _{\xi}|\hat{\kappa}(\xi ; h)|^{p} \varphi(\xi) v(\xi) .
$$

Then for all $\xi$,

$$
\lambda^{p-a}(h) \leqq|\hat{\kappa}(\xi ; h)|^{p-a}[\varphi(\xi) v(\xi)]^{1-a / p} .
$$

Let $g$ be a measurable function, and put

$$
\omega^{a}(h)=\int|g(\xi)|^{a}|\hat{\kappa}(\xi ; h)|^{a} d \xi
$$

We have

$$
\omega^{a}(h) \lambda^{\nu-a}(h) \leqq \int|\hat{\kappa}(\xi ; h)|^{p}|g(\xi)|^{a}[\varphi(\xi) v(\xi)]^{1-a / p} d \xi .
$$

Integrating $d h$ we get

$$
\int \omega^{a}(h) \lambda^{p-a}(h) d h \leqq k_{p}^{p} \int|g(\xi)|^{a}[\varphi(\xi) v(\xi)]^{1-a / p} d \xi .
$$

Since

and

$$
\int \omega^{p}(h) d h \leqq\left\{\int \omega^{a}(h) \lambda^{p-a}(h) d h\right\}^{\sigma}\left\{\int \lambda^{p}(h) d h\right\}^{1-\sigma}
$$

$$
\int \lambda^{p}(h) d h \leqq \tilde{k}_{p}^{p}\|\varphi\|^{-1}
$$

we arrive at

$$
\left\{\int \omega^{p}(h) d h\right\}^{1 / p} \leqq k_{p}^{\sigma} \tilde{k}_{p}^{1-\sigma}\left\{\int|g(\xi)|^{a}[\varphi(\xi) v(\xi)]^{1-a / p} d \xi\right\}^{1 / a}\|\varphi\|^{1 / p-1 / a}
$$


This proves that $K_{a, p} \leqq k_{p}^{\sigma} \tilde{k}^{1-\sigma} N_{a, p}$. It is obvious that $k_{p}<\infty$ for a $K$-kernel. To prove that $\tilde{k}_{p}<\infty$ we use the next.

Lemma 2.5. Suppose $\delta>0$ and $0<\epsilon \leqq 1$ are given. If $\varphi^{*}$ is decreasing then there exists a function $\rho \geqq \varphi^{*}$ such that

(i) $\rho(u) u^{1+\delta}$ is increasing,

(ii) $\rho(u) u^{1-\epsilon}$ is decreasing,

(iii)

$$
\int_{0}^{\infty} \rho(u) d u=\left(\delta^{-1}+\epsilon^{-1}\right) \int_{0}^{\infty} \varphi^{*}(v) d v
$$

Proof. Define $\rho$ by

$$
\rho(u)=\int_{0}^{\infty} \min \left\{\left(u^{-1} v\right)^{1+\delta},\left(u^{-1} v\right)^{1-\epsilon}\right\}\left[-d \varphi^{*}(v)\right] .
$$

It is obvious that $\rho$ has properties (i) and (ii). Integration by parts gives

$$
\rho(u) u=(1+\delta) \int_{0}^{u}\left(u^{-1} v\right)^{\delta} \varphi^{*}(v) d v+(1-\epsilon) \int_{u}^{\infty}\left(u^{-1} v\right)^{-\epsilon} \varphi^{*}(v) d v
$$

Property (iii) is now clear. Moreover

$$
\begin{aligned}
\rho(u) u & \geqq(1+\delta) \int_{0}^{u}\left(u^{-1} v\right)^{\delta} \varphi^{*}(v) d v \geqq(1+\delta) \int_{0}^{u}\left(u^{-1} v\right)^{\delta} \varphi^{*}(u) d u \\
& =\varphi^{*}(u) u .
\end{aligned}
$$

To complete the proof of Lemma 2.3 we note that if $\hat{\kappa}$ is a $K$-kernel then there exist $\delta>0$ and $\epsilon>0$ such that

$$
|\hat{\kappa}(\xi ; h)|^{p} \leqq v^{\delta}(\xi) v^{\delta}(h) \text { and }|\hat{\kappa}(\xi ; h)|^{p} \leqq v^{-\epsilon}(\xi) v^{-\epsilon}(h) .
$$

Given $\varphi \varepsilon \Phi$, define $\rho$ to be the function of Lemma 2.5. Then we have for all $\xi$ and $h$

$$
|\hat{\kappa}(\xi ; h)|^{p} \varphi(\xi) v(\xi) \leqq \rho\left(u^{-1}\right) u^{-1}, \quad u=v(h) .
$$

Since $\int \rho\left(u^{-1}\right) u^{-1} d u=\int \rho(u) d u \leqq\left(\delta^{-1}+\epsilon^{-1}\right)\|\varphi\|$, it follows that $\tilde{k}_{p} \leqq\left(\delta^{-1}+\right.$ $\left.\epsilon^{-1}\right)^{1 / p}<\infty$.

The quantity $k_{a}$ previously defined is the $\mathrm{L}_{1}$ norm of $|\hat{\kappa}(\theta, \cdot)|^{a} v^{-1}$ where $v(\theta)=1$. Let $\tilde{c}_{a}^{a}$ signify the ${ }_{\infty} \mathrm{L}_{1}$ norm of this function.

Lemma 2.4. $K_{a, p} \leqq k_{a}^{a / p} \tilde{c}_{a}^{1-a / p} N_{a, p}$ when $1 \leqq a \leqq p \leqq \infty$.

Proof. The quantity $\tilde{c}_{a}$ is the infimum of $\|\lambda\|^{1 / a}$ where $\lambda \varepsilon \Phi$ and

$$
|\hat{\kappa}(\xi ; h)|^{a} \leqq \lambda^{*}[v(\xi) v(h)] v(\xi) v(h) .
$$

If $f \varepsilon \mathfrak{L}_{1}$ and $f \geqq 0$, then

$$
\int|\hat{\kappa}(\xi ; h)|^{a} f[v(h)] d h \leqq \int \lambda^{*}[v(\xi) u] f(u) d u \cdot v(\xi)=\varphi(\xi) v(\xi)
$$


where $\varphi \varepsilon \Phi$ and $\|\varphi\|=\|\lambda\| \int f(u) d u$. Hence the Hölder inequality gives

$$
\begin{aligned}
\int|\hat{\kappa}(\xi ; h)|^{a} f^{1 / \tau}[v(h)] d h & \leqq\left\{|\hat{k}(\xi ; h)|^{a} d h\right\}^{1 / \sigma}\left\{\int|\hat{\kappa}(\xi ; h)|^{a} f[v(h)] d h\right\}^{1 / \tau} \\
& \leqq k_{a}^{a / \sigma}[\varphi(\xi) v(\xi)]^{1 / \tau} .
\end{aligned}
$$

Given a measurable function $g$, the quantity $K_{a, p}(g)$ is $\left\|\bar{\omega}^{a}\right\|_{\sigma}^{1 / a}$ where $\left\|\bar{\omega}^{a}\right\|_{\sigma}$ is the $\mathscr{L}_{\sigma}$ norm of the function $\bar{\omega}^{a}$ defined by

$$
\bar{\omega}^{a}[v(h)]=\int|g(\xi)|^{a}|\kappa(\xi ; h)|^{a} d \xi .
$$

Now, $\left\|\bar{\omega}^{a}\right\|_{\sigma}=\sup _{f} \int \bar{\omega}^{a}(u) f^{1 / \tau}(u) d u$ where $f \geqq 0$ and $\int f(u) d u \leqq 1$. Hence

$$
\begin{aligned}
K_{a, p}^{a}(g) & \leqq \sup _{f} \iint|g(\xi)|^{a}|\kappa(\xi ; h)|^{a} f^{1 / \tau}[v(h)] d \xi d h \\
& \leqq \sup _{\varphi} k_{a}^{a / \sigma} \int|g(\xi)|^{a}[\varphi(\xi) v(\xi)]^{1 / \tau} d \xi
\end{aligned}
$$

where the supremum is taken over $\varphi \varepsilon \Phi$ with $\|\varphi\| \leqq\|\lambda\|$. In other words, $K_{a, p}(g) \leqq k_{a}^{1 / \sigma}\|\lambda\|^{1 / a r} N_{a, p}(g)$.

3. Bernstein's theorem. The most general and precise statements relating Lipschitz conditions to integrability of the Fourier transform are summarized in the next.

Theorem 1. Let $1 \leqq a \leqq \infty$ and $1 \leqq p \leqq \infty$ be given, and put $\alpha=n / p-n / a^{\prime}$.

(i) If $1 \leqq a \leqq 2$ then the Fourier transform maps $\boldsymbol{\Lambda}_{a, p}^{\alpha}$ continuously into ${ }_{a}, \mathrm{~L}_{p}$.

(ii) If $2 \leqq a \leqq \infty$ then the Fourier transform maps ${ }_{a}, \mathbf{L}_{p}$ continuously into $\mathbf{\Lambda}_{a, p}^{\alpha}$ when $p<\infty$.

Corollary. For $1 \leqq p<\infty$ and $\alpha=n / p-n / 2$, the Fourier transform establishes an equivalence between the Banach spaces $\mathbf{\Lambda}_{2, p}^{\alpha}$ and ${ }_{2} \mathbf{L}_{p}$.

The case $p=1$ (and $n=1$ ) of this corollary is Beurling's precise version [1] of Bernstein's Theorem.

The statement of Theorem 1 is most interesting when $\alpha \geqq 0$; so $a^{\prime} \geqq p$ and ${ }_{a}, \mathbf{L}_{p}$ is a subspace of $\mathbf{L}_{p}$. When $\alpha \leqq 0$ the theorem is not so useful for applications, but it does offer the technical convenience of showing, as we shall see later, that statements (i) and (ii) are duals of each other.

Theorem 1 is a specialization of the following pair of dual statements.

Proposition 3.1. For $1 \leqq a \leqq 2,1 \leqq p \leqq \infty$, and all real $\alpha$, the Fourier transform maps $\mathbf{\Lambda}_{a, p}^{\alpha}$ continuously into $\mathbf{K}_{a^{\prime}, p}^{\alpha}$.

Proposition 3.1'. For $1 \leqq a \leqq 2,1 \leqq p \leqq \infty$, and all real $\alpha$, if $g \in \mathbf{K}_{a, p}^{\alpha}$ then for the Fourier transform $g$ we have $\Lambda_{a^{\prime}, p}^{\alpha}(\hat{g}) \leqq K_{a, p}^{\alpha}(g)$ when the norms are suitably normalized. 
When more definitions are given and more machinery is developed it will be apparent that the general statements are consequences of Proposition 3.1 or $3.1^{\prime}$ for one particular choice of $\alpha$. Nevertheless, what follows is sufficient for the cases $1 \leqq p<a^{\prime}$ of Theorem 1 without further ado.

Proofs of Proposition 3.1 and 3.1'. Suppose $0<\alpha<k$, and put

$$
\hat{\kappa}(\xi ; h)=b_{n}^{-\alpha / n}|h|^{-\alpha}|\xi|^{-\alpha}\left(e^{2 \pi i \xi \cdot h}-1\right)^{k} .
$$

Then clearly $\hat{\kappa}$ is a $K$-kernel and we fix the $\mathbf{K}_{a, p}^{\alpha}$-norm by

$$
K_{a, p}^{\alpha}(g)=\left\{\int\left(\int\left|g(\xi) v^{\alpha / n}(\xi) \dot{\hat{\kappa}}(\xi ; h)\right|^{a} d \xi\right)^{p / a} d h\right\}^{1 / p},
$$

while the $\boldsymbol{\Lambda}_{a, \nu}^{\alpha}$-norm is normalized by

$$
\Lambda_{a, p}^{\alpha}(f)=\&_{p} \text {-norm of }|h|^{-\alpha}\left\|\Delta^{k}(h) f\right\|_{a} .
$$

If $f(x) \leftrightarrow g(\xi)$ is a Fourier transform pair then so is

$$
|h|^{-\alpha} \Delta^{k}(h) f(x) \leftrightarrow g(\xi) v^{\alpha / n}(\xi) \hat{\kappa}(\xi ; h) .
$$

Hence if $g \varepsilon \mathbf{K}_{a, p}^{\alpha}$ with $1 \leqq a \leqq 2$,

$$
|h|^{-\alpha}\left\|\Delta^{k}(h) f\right\|_{a^{\prime}} \leqq\left\|g v^{\alpha / n} \hat{\kappa}(\cdot, h)\right\|_{a} .
$$

Therefore $\Lambda_{a^{\prime}, p}^{\alpha}(f) \leqq K_{a, p}^{\alpha}(g)$. If $g \varepsilon \mathcal{O}$ then

$$
|h|^{-\alpha}\left\|\Delta^{k}(h) f\right\|_{a} \geqq\left\|g v^{\alpha / n} \hat{k}(\cdot, h)\right\|_{a^{\prime}},
$$

so $\Lambda_{a, p}^{\alpha}(f) \geqq K_{a^{\prime}, p}^{\alpha}(g)$. This inequality persists as long as $1 \leqq a \leqq 2$ and $g$ is a function, even if $f \notin \boldsymbol{\Lambda}_{a, p}^{\alpha}$.

In contrast to the pair of dual Propositions 3.1 and $3.1^{\prime}$ there is the self-dual generalization of the classical theorems of Hausdorff, Young, and Paley.

Proposition 3.2. For $1<a<2$ and $1 \leqq p \leqq \infty$ the Fourier transform maps $\mathbf{L}_{a, p}$ into $\mathbf{L}_{a^{\prime}, p}$.

Proof. Apply the Marcinkiewicz Interpolation Theorem using the obvious fact that the Fourier transform takes $L_{1}$ to $L_{\infty}$ and the Plancherel Theorem which says that the Fourier transform maps $\mathbf{L}_{2}$ to $\mathbf{L}_{2}$. For the Marcinkiewicz Interpolation Theorem see [5].

Proposition 3.2 is a best possible result. A partial converse with dimensional restrictions is

Proposition 3.3. Suppose $f(x)$ is a decreasing function of $|x|$. If $f \in \varepsilon \mathbf{L}_{a, p}$ where $1<a<\nu=2 n /(n-1)$ then $\hat{f} \varepsilon \mathbf{L}_{a^{\prime}, p}$. If $f \varepsilon \mathbf{L}_{\nu, 1}$ then $\hat{f} \varepsilon \mathbf{L}_{\nu^{\prime}, \infty}$. (In case $n=1$ the last is to be interpreted as: if $f(x)$ is bounded and decreases to 0 as $|x| \rightarrow \infty$ then $\hat{f}$ belongs to weak $L_{1}$ ).

Proof. As long as $1<a<\nu$ we can find $b$ and $\beta$ such that $1 \leqq b \leqq 2,0<$ $\beta<1 / b$, and $1 / b-\beta / n=1 / a$. In these circumstances we have, according to 
Proposition 1.8, that $f \varepsilon \mathbf{\Lambda}_{b, p}^{\beta}$. Proposition 3.1 applies to show that $\hat{f} \varepsilon \mathbf{K}_{b, p}^{\beta}$; and $\mathrm{K}_{b, \nu}^{\beta} \subset \mathrm{L}_{a^{\prime}, p}$ by Proposition 2.6. In the limiting case $a=\nu$ we use the fact that if $f \varepsilon \mathrm{L}_{\nu, p}$ is decreasing then $\Lambda_{2, \infty}^{1 / 2}(f)<\infty$; so $\hat{f} \varepsilon \mathbf{K}_{2, \infty}^{1 / 2} \subset \mathbf{L}_{\nu^{\prime}, \infty}$.

Corollary. For $1<a<\infty, 1 \leqq p \leqq \infty$, if $g \varepsilon \mathrm{L}_{a, p}$ then there exists $f \varepsilon \mathbf{L}_{a^{\prime}, p}$ such that $\hat{\mathrm{f}}$ is equimeasurable with $|g|$.

Proof. Put $f\left(x_{1}, x_{2}, \cdots, x_{n}\right)=\gamma\left(x_{1}\right) \hat{\chi}\left(x_{2}\right) \cdots \hat{\chi}\left(x_{n}\right)$ where $\hat{\chi}(u)=(\sin \pi u) / \pi u$ and

$$
\gamma(u)=\int_{0}^{\infty} \cos \pi u v g^{*}(v) d v .
$$

By the 1-dimensional version of Proposition 3.3 it follows that $\gamma \varepsilon \mathrm{L}_{a^{\prime}, p}$; hence $f \varepsilon \mathrm{L}_{a^{\prime}, p}$. On the other hand

$$
\hat{f}\left(\xi_{1}, \xi_{2}, \cdots \xi_{n}\right)=g^{*}\left(2\left|\xi_{1}\right|\right) \chi\left(\xi_{2}\right) \cdots \chi\left(\xi_{n}\right)
$$

where $\chi(t)=1$ if $-\frac{1}{2}<t<\frac{1}{2}$ and $\chi(t)=0$ if $|t|>\frac{1}{2}$. Therefore $\hat{f}^{*}=g^{*}$, i.e. $\hat{f}$ is equimeasurable with $|g|$.

Proposition 3.2 cannot be extended to the case $a=2$. The limitations are most clearly apparent for lacunary functions as defined in \$2. Our treatment of lacunary Fourier transforms is based on a new device and seems to differ markedly from classical methods.

Lemma 3.1. For $1<a \leqq 2$ the Fourier transform maps $\mathbf{L}_{a}$ into $\mathbf{K}_{a^{\prime}, 2}$.

Proof. We appeal to Proposition 8.3 which says that $\mathbf{L}_{a} \subset \mathbf{\Lambda}_{a, 2}^{0}$ for $1<a \leqq 2$. The result then follows from Proposition 3.1 with $\alpha=0$.

Proposition 3.4. Suppose $1 \leqq a \leqq 2$. If $f \in \mathrm{L}_{a}$ has a lacunary Fourier transform then $f \varepsilon \mathbf{L}_{b}$ for all $b, a \leqq b<\infty$.

Proof. Suppose $1<a \leqq 2$ and $2 \leqq b<\infty$. If $f \varepsilon \mathbf{L}_{a}$ then $\hat{f} \varepsilon \mathbf{K}_{a^{\prime}, 2}$ by Lemma 3.1. Since $\hat{f}$ is lacunary it follows from Proposition 2.8 that $\hat{f} \varepsilon \mathbf{K}_{b^{\prime}, 2}$. The dual of Lemma 3.1 furnishes the conclusion that $f \varepsilon \mathbf{L}_{b}$. The case $a=1$ requires a special argument. Let $E$ be a lacunary set. We have just proved that there exists a constant $M_{E}$ such that if $g \varepsilon \mathrm{L}_{3 / 2}$ and $\hat{g}=0$ almost everywhere outside $E$ then $\|g\|_{2}<M_{E}\|g\|_{3 / 2}$. Now suppose $f \varepsilon L_{1}$ and $\hat{f}(\xi)=0$ for $\xi \notin E$. Let $u \varepsilon \mathbf{L}_{1} \cap \mathbf{L}_{2}$ be arbitrary and put $g=u * f$. Then $g \varepsilon \mathbf{L}_{1} \cap \mathbf{L}_{2} \subset \mathbf{L}_{3 / 2}$; so we have two estimations:

$$
\|g\|_{3 / 2} \leqq\|g\|_{1}^{1 / 2}\|g\|_{2}^{1 / 2}, \quad\|g\|_{2} \leqq M_{E}\|g\|_{3 / 2} .
$$

It follows that $\|g\|_{3 / 2} \leqq M_{E}\|g\|_{1}$. Choosing a family $\{u\}$ which gives an approximate identity on $\mathrm{L}_{1}$ and $\mathrm{L}_{2}$, we get $f \varepsilon \mathrm{L}_{3 / 2}$.

The negative statement for the case $a=2$ of Proposition 3.2 is

Proposition 3.5. The Fourier transform maps $\mathrm{L}_{2, p}$ continuously into $\mathrm{L}_{2, a}$ iff $p \leqq 2 \leqq q$. More emphatically, the Fourier transform maps $\mathbf{L}_{1} \cap \mathbf{C}_{0}$ into $\mathbf{L}_{2, a}$ iff $q \geqq 2$. 
Proof. If the Fourier transform maps $\mathbf{L}_{2, p}$ into $\mathbf{L}_{2, q}$ then by duality it maps $\mathbf{L}_{2, q^{\prime}}$ into $\mathbf{L}_{2, p^{\prime}}$. It is therefore enough to show that $\mathbf{L}_{2,1}$ does not map into $\mathbf{L}_{2, a}$ for any $q<2$. Choose $u \varepsilon D$ with $u(\xi)=0$ for $|\xi|>\frac{1}{2}$, and choose a unit vector $\theta$. Put $g(\xi)=\sum_{1}^{\infty} c_{j} u\left(\xi+2^{i} \theta\right)$ where $\left\{c_{j}\right\}$ is any sequence such that $\sum\left|c_{i}\right|^{2}<\infty$. Then $g$ is lacunary, and $g$ together with all the derivatives belongs to $\mathbf{L}_{2}$. Hence $f=\hat{g} \varepsilon \mathbf{L}_{2}$. By Proposition 3.4, $f \varepsilon \mathbf{L}_{b}$ for all $b, 1 \leqq b<\infty$, in particular, $f \varepsilon \mathbf{L}_{2,1}$, but, say if $c_{j}^{-2}=j \log (j+2) \log \log ^{2}(j+2), g$ does not belong to $L_{2, a}$ for any $q<2$. The $f$ we have constructed is not necessarily continuous, but if $c_{j}= \pm\left|c_{j}\right|$ with a toss of a coin determining the sign, then with probability $1, f \varepsilon \mathbf{C}_{0}$.

4. The fundamental inversion formula. We shall presently define a class $I_{k}$ of kernels such that the following holds.

Theorem 2. For each $f \varepsilon \mathbf{\Lambda}_{a, 1}^{\alpha}$, where $0<\alpha<k$ and $a \alpha=n$, in particular for each $f \varepsilon \hat{\mathcal{\theta}}$, we have an absolutely convergent integral representation

whenever $\kappa \varepsilon I_{k}$.

$$
f(x)=\iint \kappa(x-y ; h) \Delta^{k}(h) f(y) d y d h
$$

Remark. This formula enables one to recover $f$ from $\Delta^{k}(h)$. It is similar to a formula of Calderón [2; $\$ 14.2]$, but it is basically simpler.

The class $I_{k}$ consists of the function $\kappa$ defined on $R^{n} \times R^{n}$ with the properties:

(a) $\kappa(T x ; h)=|\operatorname{det} T| \kappa\left(x ; T^{-1} h\right)$ for any linear transformation $T$ which is a dilation of an orthogonal transformation.

(b) when $h \neq 0, \kappa(\cdot ; h) \varepsilon \mathbf{L}_{1}$ and $\hat{\kappa}(\cdot ; h) \varepsilon \mathbf{L}_{1}$ where $\hat{\kappa}(\cdot ; h)$ is the Fourier transform of $\kappa(\cdot ; h)$.

$\left(c_{k}\right)$ for some $\theta \neq 0$,

$$
\int\left(e^{2 \pi i \theta h}-1\right)^{k} \hat{\kappa}(\theta ; h) d h=1 .
$$

The classes $I_{k}$ are quite rich. The condition $\left(c_{k}\right)$ is essentially a normalizing condition. Thus, for a suitable constant $c_{k}$,

$$
\hat{\kappa}(\xi ; h)=c_{k}\left(e^{-2 \pi i \xi h}-1\right)^{k} g\left(|\xi|^{2}|h|^{2}\right)
$$

gives a kernel $\kappa \varepsilon I_{k}$ whenever $g$ is a sufficiently differentiable, positive decreasing function on $(0, \infty)$ such that $\int_{0}^{\infty} g(t) t^{n / 2} d t<\infty$. We shall make no use of the generality available for the class $I_{k}$. We shall only need the existence of a function $\hat{\kappa} \varepsilon \mathcal{O}$ which is radial, i.e., $\hat{\kappa}(\xi)$ depends only on $|\xi|$, such that

$$
\int\left(e^{2 \pi i \theta h}-1\right)^{k} \hat{\kappa}(h) d h=1
$$

for some $\theta$ with $|\theta|=1$. For such a $\hat{\kappa}$,

$$
\kappa(\xi ; h)=|h|^{-n} \kappa\left(|h|^{-1} \xi\right)=\kappa_{|h|}(\xi)
$$

is a kernel in $I_{k}$. 
Proof of Theorem 2. It follows from Property (a) that

$$
\|\kappa(\cdot ; h)\|_{a^{\prime}}=M_{a^{\prime}}|h|^{-n / a}
$$

where, by Property (b), the constant $M_{a^{\prime}}$ is finite for all $a, 1 \leqq a \leqq \infty$. Therefore

$$
\begin{aligned}
\left|\int \kappa(x-y ; h) \Delta^{k}(h) f(y) d y\right| & \leqq\|\kappa(. ; h)\|_{a^{\prime}}\left\|\Delta^{k}(h) f\right\|_{a} \\
& \leqq M_{\imath^{\prime}}|h|^{-n / a}\left\|\Delta^{k}(h) f\right\|_{a} .
\end{aligned}
$$

Hence the double integral

$$
g(x)=\iint \kappa(x-y ; h) \Delta^{k}(h) f(y) d y d h
$$

is absolutely convergent when $f \varepsilon \mathbf{\Lambda}_{a, 1}^{\alpha}$ and $\alpha=n / a$. Moreover $\|g\|_{\infty} \leqq M_{a}, \Lambda_{a, 1}^{\alpha}(f)$. It remains only to show that $g(0)=f(0)$ for $f \varepsilon \hat{\mathcal{\theta}}$. Taking Fourier transforms we have

$$
g(0)=\iint \hat{\kappa}(\xi ; h)\left(e^{2 \pi i \xi h}-1\right)^{k} \hat{f}(\xi) d \xi d h .
$$

Since $\hat{f} \varepsilon \theta$, the variable $\xi$ is bounded away from 0 and $\infty$. Also

$$
\int\left|e^{2 \pi i \xi h}-1\right|^{k}|\hat{\kappa}(\xi ; h)| d h
$$

is a finite quantity which does not depend on $\xi \neq 0$. Thus the double integral for $g(0)$ is absolutely convergent, and we may interchange the order of integration. Since

$$
\int\left(e^{2 \pi i \xi h}-1\right)^{k} \hat{\kappa}(\xi ; h) d h=1
$$

for all $\xi$, we get

$$
g(0)=\int \hat{f}(\xi) d \xi=f(0) .
$$

Note, in passing, that we have proved $\|f\|_{\infty} \leqq M_{a^{\prime}} \Lambda_{a, 1}^{n / a}(f)$. This establishes Proposition 1.2(i).

Restricting our attention to radial kernels $\kappa$, we can make some simplifications. Given $t>0$, put $\bar{\Delta}^{k}(t) f(x)$ for the mean value of $\Delta^{k}(h) f(x)$ over the sphere $|h|=t$. The inversion formula then reads

$$
f(x)=\iint \kappa_{t}(x-y) \bar{\Delta}^{k}(t) f(y) d y d t
$$

where the integration in $t$ is extended over $(0, \infty)$ and $d t=t^{-1} d t$.

5. The basic inclusion relations for Lipschitz spaces. The main result we want here is 
Theorem 3. Suppose $\beta \leqq \alpha, 1 / a-\alpha / n=1 / b-\beta / n$, and $p \leqq q$. Then $\mathbf{\Lambda}_{a, p}^{\alpha} \subset \mathbf{\Lambda}_{b, a}^{\beta}$.

The proof of Theorem 3 depends upon a fact of great importance in its own right. Let $\lambda \varepsilon \hat{\Theta}$ be a radial function. Given $u$ a continuous function of compact support in $R^{n} \times R_{+}$define $T^{\alpha}(\lambda) u$ by

$$
T^{\alpha}(\lambda) u(x)=\iint t^{\alpha} \lambda_{t}(x-y) u(y ; t) d y d t .
$$

Then $T^{\alpha}(\lambda) u \varepsilon \hat{\mathcal{\theta}}$. The essential result is

Proposition 5.1. Suppose $\beta \leqq \alpha, 1 / a-\alpha / n=1 / b-\beta / n$, and $p \leqq q$. Then given a radial function $\lambda \varepsilon \|, T^{\alpha}(\lambda)$ extends to a bounded linear transformation $T^{\alpha}(\lambda): \mathfrak{L}_{p}\left(\mathbf{L}_{a}\right) \rightarrow \boldsymbol{\Lambda}_{b, a}^{\beta}$.

Proof. Write $f=T^{\alpha}(\lambda) u$. Then $f \varepsilon \hat{\Theta}$ and

$$
|h|^{-\beta} \Delta^{j}(h) f(x)=\iint|h|^{-\beta} t^{\alpha} \Delta^{i}(h) \lambda_{t}(y) u(x-y ; t) d y d t .
$$

Put $\delta(h)=|h|^{-\beta}\left\|\Delta^{i}(h) f\right\|_{b}, \psi(t)=\|u(\cdot ; t)\|_{a}$, and define $c$ by $1 / a-1 / b=$ $1 / c^{\prime}$. Young's Theorem says that $\mathrm{L}_{c} * \mathrm{~L}_{a} \subset \mathrm{L}_{b}$, and the norm inequality gives, in particular,

$$
\delta(h) \leqq \int|h|^{-\beta} t^{\alpha}\left\|\Delta^{i}(h) \lambda_{t}\right\|_{c} \psi(t) d t .
$$

Now $\Delta^{i}(h) \lambda_{t}=\left(\Delta^{i}\left(t^{-1} h\right) \lambda\right)_{t}$, and for any $g \varepsilon \mathbf{L}_{c}$ we have $\left\|g_{t}\right\|_{c}=t^{-n / c^{\prime}}\|g\|_{0}$. Hence, given the supposed relations between $a, b, c, \alpha$, and $\beta$, it follows that $|h|^{-\beta} t^{\alpha}\left\|\Delta^{i}(h) \lambda_{t}\right\|_{c}=\varphi\left(t^{-1} h\right)$ where $\varphi(h)=|h|^{-\beta}\left\|\Delta^{i}(h) \lambda\right\|_{c}$. Thus one has

$$
\delta(h)=\int \varphi\left(t^{-1} h\right) \psi(t) d t .
$$

This is a convolution over the multiplicative group $R_{+}$(all functions of $h$ depend only on $|h|$ here); applying Young's Theorem again we get $\|\delta\|_{a} \leqq\|\varphi\|_{r}\|\psi\|_{p}$ where $1 / p-1 / q=1 / r^{\prime}$, or

$$
\Lambda_{b, q}^{\beta}(f) \leqq \Lambda_{c, r}^{\beta}(\lambda)\|u\|_{a, p} .
$$

Remark. The proof of Proposition 5.1 has assumed $\beta>0$, but, as soon as the $\Lambda_{b, a}^{\beta}$-norms are defined for $\beta \leqq 0$, it will be obvious that the statements persist for all real $\beta$.

Proof of Theorem 3. Given $f \varepsilon \hat{\mathcal{\theta}}$, put $D^{\alpha} f(x ; h)=|h|^{-\alpha} \Delta^{k}(h) f(x)$ and $\bar{D}^{\alpha} f(x ; t)$ $=t^{-\alpha} \overline{\Delta^{k}}(t) f(x)$. Then $\left\|\bar{D}^{\alpha} f\right\|_{a, p} \leqq\left\|D^{\alpha} f\right\|_{a, p}=\Lambda_{a, p}^{\alpha}(f)$. Thus $\bar{D}^{\alpha}$ extends to a bounded linear transformation $\bar{D}^{\alpha}: \mathbf{\Lambda}_{a, p}^{\alpha} \rightarrow \mathfrak{L}_{p}\left(\mathbf{L}_{a}\right)$. According to Theorem 2, $T^{\alpha}(\kappa) \circ \bar{D}^{\alpha}=$ identity on $\hat{\mathcal{G}}$, where $\kappa$ is the kernel of the inversion formula. Therefore, by Proposition 5.1, $T^{\alpha}(\kappa) \circ \bar{D}^{\alpha}$ gives an inclusion map of $\mathbf{\Lambda}_{a, p}^{\alpha}$ in $\mathbf{\Lambda}_{b, a}^{\beta}$. 
Remark. We have proved in passing that the norm $\left\|\bar{D}^{\alpha} f\right\|_{a, p}$ is equivalent to the norm $\Lambda_{a, p}^{\alpha}(f)$.

Proposition 5.1 gives a bounded linear transformation $T^{\alpha}(\lambda): \mathfrak{L}_{p}\left(\mathbf{L}_{a}\right) \rightarrow \mathbf{\Lambda}_{a, p}^{\alpha}$. We shall now construct the formal adjoint. For $f \varepsilon \hat{\theta}$ define $T_{\alpha}(\lambda) f$ by

$$
T_{\alpha}(\lambda) f(x ; t)=\int t^{\alpha} \lambda_{t}(x-y) f(y) d y .
$$

Proposition 5.2. Let $\lambda \varepsilon \hat{O}$ be a radial function. Then $T_{-\alpha}(\lambda)$ extends to $a$ bounded linear transformation $T_{-\alpha}(\lambda): \mathbf{\Lambda}_{a, p}^{\alpha} \rightarrow \mathscr{L}_{p}\left(\mathbf{L}_{a}\right)$.

Proof. Suppose $0<\alpha<1$. Put $u=T_{-\alpha}(\lambda) f$. Then

$$
u(x ; t)=\int t^{-\alpha} \lambda_{t}(y)\{f(x-y)-f(x)\} d y
$$

since $\int \lambda_{t}(y) d y=0$. We may rewrite this as

$$
u(x ; t)=\int\left(t|y|^{-1}\right)^{-n-\alpha} \lambda\left(t^{-1} y\right)|y|^{-\alpha} \Delta(-y) f(x) d y \cdot n b_{n} .
$$

It follows that

$$
\|u(\cdot ; t)\|_{a} \leqq \int \varphi\left(t|y|^{-1}\right) \psi(|y|) d y
$$

where $\varphi(t)=n b_{n} t^{-n-\alpha} \lambda\left(t^{-1}\right)$ and $\psi(|y|)=|y|^{-\alpha}\|\Delta(y) f\|_{a}$. Once again we have a convolution on $R_{+}$and Young's Theorem gives

$$
\|u\|_{a, p} \leqq\|\varphi\|_{1}\|\psi\|_{p}=\text { const. } \Lambda_{a, p}^{\alpha}(f) .
$$

The proof is valid for $0<\alpha<2$ since we may write

$$
u(x ; t)=\frac{1}{2} \int t^{-\alpha} \lambda_{t}(y)\{f(x+y)+f(x-y)-2 f(x)\} d y
$$

On the other hand, we shall soon see that the truth of the statement for a single value of $\alpha$ implies its truth for all values of $\alpha$.

The fundamental inversion formula is $T^{\alpha}(\kappa) \circ D^{\alpha}=I$ where $I$ denotes some extension of the identity on $\hat{\mathcal{O}}$. It is sometimes convenient to make use of a general class of inversion formulas given by

Proposition 5.3. Given a non-trivial radial function $\lambda \varepsilon \hat{\mathcal{O}}$ there exists a constant $c_{\lambda}>0$ such that for all $\alpha, T^{\alpha}(\bar{\lambda}) \circ T_{-\alpha}(\lambda)=c_{\lambda} I$.

Proof. Take $f \varepsilon \hat{\mathcal{O}}$ and put $u=T_{-\alpha}(\lambda) f$. Then for the Fourier transforms (in the space variables) we have

Also, if $g=T^{\alpha}(\bar{\lambda}) u$ then

$$
\hat{u}(\xi ; t)=t^{-\alpha} \lambda(t \xi) \hat{f}(\xi) .
$$

$$
\hat{g}(\xi)=\int t^{\alpha} \bar{\lambda}(t \xi) \hat{u}(\xi ; t) d t .
$$


The end formula is

$$
\hat{g}(\xi)=\int|\lambda(t \xi)|^{2} \hat{f}(\xi) d t=c_{\lambda} \hat{f}(\xi)
$$

where $c_{\lambda}=\int|\lambda(t)|^{2} d t$.

For the spaces $\boldsymbol{\Lambda}_{a, p}^{\alpha}$ the Lipschitz index $\alpha$ is closely bound together with the Lebesque index $a$, and we do not obtain a larger space merely by lowering the Lipschitz index. If, however, some supplementary growth condition is imposed then such inclusion relations are available.

Proposition 5.4. Suppose $1 / a-\alpha / n<1 / b-\beta / n<1 / c$. Under either of the conditions

(i) $\max (a, c) \leqq b$,

(ii) $\min (a, c)<b$ and $\left|a^{-1}-c^{-1}\right| \beta>\left|b^{-1}-c^{-1}\right| \alpha$, we have $\mathbf{\Lambda}_{a, p}^{\alpha} \cap \mathbf{L}_{c} \subset \mathbf{\Lambda}_{b, a}^{\beta}$.

Corollary. If $0<\beta<\alpha$ and $\mathbf{1} \leqq c \leqq a \leqq \infty$ then $\mathbf{\Lambda}_{a, \infty}^{\alpha} \cap \mathbf{L}_{c} \subset \mathbf{\Lambda}_{a, 1}^{\beta}$.

The materials for the proof of Proposition 5.4 have already been assembled, but it will be convenient to postpone the demonstration until after Theorem $2^{*}$ in the next section.

6. Riesz potentials. Let $\rho$ be a function defined on $R^{n}-\{0\}$ which is nonvanishing, infinitely differentiable, and homogeneous of degree 1 . A canonical choice for $\rho$ in $\rho(\xi)=2 \pi|\xi|$, but this is not critical for what follows.

The Riesz potential of order $\alpha$ is defined as the mapping

$$
R^{\alpha}: \hat{\vartheta} \rightarrow \hat{\mathcal{O}} \text { given by } \widehat{R^{\alpha} f}=\rho^{-\alpha} \hat{f}
$$

It is clear that $R^{\alpha}$ is a bijection for all real $\alpha$. Moreover, $R^{\alpha} R^{\beta}=R^{\alpha+\beta}$ and $R^{0}$ is the identity.

We also use the symbol $R^{\alpha}$ for any unique closed extension. The relations between Riesz potentials and the operations of Proposition 5.1 and 5.2 is important.

Lemma 6.1. Let $\lambda \varepsilon \hat{\vartheta}$ be a radial function. Then

$$
R^{\beta} \circ T^{\alpha}(\lambda)=T^{\alpha+\beta}\left(R^{\beta} \lambda\right) \text { and } T_{\alpha}(\lambda) \circ R^{\beta}=T_{\alpha+\beta}\left(R^{\beta} \lambda\right) .
$$

Proof. Elementary computation.

The importance of the Riesz potentials in the theory of Lipschitz spaces follows from

Proposition 6.1. $\quad R^{\beta} \Lambda_{a, p}^{\alpha}=\Lambda_{a, p}^{\alpha+\beta}$.

Proof. According to Theorem 2 and Proposition 5.1, each $f \varepsilon \mathbf{\Lambda}_{a, p}^{\alpha}$ can be written as $f=T^{\alpha}(\kappa) u$ where $\kappa$ is the kernel of the inversion formula and $u \varepsilon$ $\mathscr{L}_{p}\left(\mathbf{L}_{c}\right)$. Therefore

$$
R^{\beta} f=R^{\beta} T^{\alpha}(\kappa) u=T^{\alpha+\beta}\left(R_{\kappa}^{\beta}\right) u
$$


Using Proposition 5.1 again, we get $R^{\beta} f \varepsilon \mathbf{\Lambda}_{a, p}^{\alpha+\beta}$. This proves that $R^{\beta} \boldsymbol{\Lambda}_{a, p}^{\alpha} \subset \mathbf{\Lambda}_{a, p}^{\alpha+\beta}$, but then also $R^{-\beta} \boldsymbol{\Lambda}_{a, p}^{\alpha+\beta} \subset \mathbf{\Lambda}_{a, p}^{\alpha}$ and $R^{-\beta} R^{\beta}=R^{\beta} R^{-\beta}=I$.

Proposition 6.1 has been given a meaning when $\alpha>0$ and $\alpha+\beta>0$.

Definition. $\quad \boldsymbol{\Lambda}_{a, p}^{\alpha}$ is defined for $\alpha \leqq 0$ so that

$$
R^{\beta} \boldsymbol{\Lambda}_{a, p}^{\alpha}=\mathbf{\Lambda}_{a, p}^{\alpha+\beta}
$$

holds for all real $\alpha$ and $\beta$.

In particular, we have Lap $\mathbf{\Lambda}_{a, p}^{\alpha}=\mathbf{\Lambda}_{a, p}^{\alpha-2}$ where Lap $=R^{-2}$ is the Laplacian. More generally, one can see how the Lipschitz spaces can be defined as the completion of $\hat{\Theta}$ for various norms based on combinations of difference operators and homogeneous differential operators with constant coefficients.

There is now no difficulty in filling the gaps where proofs of general statements were given for only a restricted range of $\alpha$. In addition, Theorem 2 can be given a more general form. Suppose $\kappa \varepsilon \hat{\mathcal{O}}$ is a non-trivial radial function. Put

$$
\Delta_{\kappa}(t) f=c_{\kappa}^{-1} \kappa_{t} * f
$$

where $c_{\kappa}$ is the constant of Proposition 5.3. Observe that for each $t>0, \Delta_{\kappa}(t)$ gives a linear transformation of $\hat{\mathcal{O}}$ into itself.

Theorem 2*. For each non-trivial radial function $\kappa \varepsilon \hat{\mathcal{O}}$ there exists a family of linear operations $\Delta_{\kappa}(t)$ defined for $t>0$ such that for each $f \varepsilon \mathbf{\Lambda}_{\infty, 1}^{0}$

$$
f(x)=\iint \kappa_{t}(x-y) \Delta_{\kappa}(t) f(y) d y d t
$$

with the double integral being absolutely convergent, and

$$
\Lambda_{a, p}^{\alpha}(f) \approx\left\{\int\left(t^{-\alpha}\left\|\Delta_{\kappa}(t) f\right\|_{a}\right)^{p} d t\right\}^{1 / p}
$$

where $\approx$ denotes equivalence of norms, the quantities being finite when $-\infty<$ $\alpha<\infty, 1 \leqq a \leqq \infty, 1 \leqq p \leqq \infty$, and $f \varepsilon \hat{\mathcal{O}}$.

Proof. This statement is simply a summary of parts of Proposition 5.1, 5.2, and 5.3.

The definition of the $\mathbf{\Lambda}_{a, p}^{\alpha}$-norm given by Theorem $2^{*}$ is technically very convenient, and it has the advantage that there is a great deal of freedom in the choice of the kernel $\kappa$. This is illustrated in

Proof of Proposition 5.4. We wish to show that, under suitable hypotheses, $\mathbf{\Lambda}_{a, \infty}^{\alpha} \cap \mathbf{L}_{c} \subset \mathbf{\Lambda}_{b, 1}^{\beta}$. To do this, let the $\mathbf{\Lambda}_{a, \infty}^{\alpha}$-norm be defined using a kernel $\kappa$, and define the $\boldsymbol{\Lambda}_{b, 1}^{\beta}$-norm using the kernel $\lambda=\kappa^{*} * \kappa$. (Note that $\lambda_{t}=\kappa_{t} * \kappa_{t}$.) We also use the general inequality

$$
\left\|u_{t} * g\right\|_{a} \leqq t^{n(1 / q-1 / p)}\|u\|_{r}\|g\|_{p}
$$

where $1 / p-1 / q=1-1 / r$. If $f \varepsilon \mathbf{\Lambda}_{a, \infty}^{\alpha}$ then $\left\|\kappa_{t} * f\right\|_{a}=O\left(t^{\alpha}\right)$; so

$$
\left\|\lambda_{t} * f\right\|_{\bar{a}}=\left\|\kappa_{t} *\left(\kappa_{t} * f\right)\right\|_{\bar{a}}=O\left(t^{\bar{\alpha}}\right)
$$


whenever $a \leqq \bar{a}$ and $\bar{\alpha}=\alpha+n(1 / a-1 / \bar{a})$. We also have for $f \varepsilon \mathbf{L}_{c}$,

$$
\left\|\lambda_{t} * f\right\|_{\bar{c}}=O\left(t^{\bar{\gamma}}\right) \text { whenever } c \leqq \bar{c} \text { and } \bar{\gamma}=-n(1 / c-1 / \bar{c}) \text {. }
$$

To prove that $f \varepsilon \mathbf{\Lambda}_{b, 1}^{\beta}$ we must show that $A<\infty$ and $C<\infty$ where

$$
A=\int_{0}^{1} t^{-\beta}\left\|\lambda_{t} * f\right\|_{b} d t \text { and } C=\int_{1}^{\infty} t^{-\beta}\left\|\lambda_{t}+f\right\|_{b} d t .
$$

Suppose $a \leqq b$, then choosing $\vec{a}=b$ in the above estimate we get $t^{-\beta}\left\|\lambda_{t} * f\right\|_{b}=$ $O\left(t^{\delta}\right)$ where $\delta=(1 / b-\beta / n)-(1 / a-\alpha / n)$. Hence if $a \leqq b$ and $\delta>0$, then $A<\infty$. Similarly, if $c \leqq b$ we have $t^{-\beta}\left\|\lambda_{t} * f\right\|_{b}=O\left(t^{-\epsilon}\right)$ where $\epsilon=1 / c-$ $(1 / b-\beta / n)$. Therefore $c \leqq b$ and $\epsilon>0$ imply $C<\infty$. Proposition 5.4 is demonstrated for the hypotheses (i). If, say, $a<b<c$, we need a new argument to prove that $C<\infty$. Taking $\bar{a}=a, \bar{c}=c$ and interpolating we get $t^{-\beta}\left\|\lambda_{t} * f\right\|_{b}=$ $O\left(t^{\sigma \alpha-\beta}\right)$ where $1 / b=\sigma / a+(1-\sigma) / c$. Hypothesis (ii) guarantees $\sigma \alpha<\beta$. The case $c<b<a$ is similar.

7. Duality and interpolation. The chief result about the spaces $\mathbf{\Lambda}_{a, p}^{\alpha}$ with $\alpha<0$ is contained in

Proposition 7.1. For $f \varepsilon \hat{\mathcal{O}}$, the norm

$$
\|f\|=\sup \left\{\left|\int f(x) g(x) d x\right|: \Lambda_{a, p}^{\alpha}(g) \leqq 1\right\}
$$

is equivalent to $\Lambda_{a^{\prime}, p^{\prime}}^{-\alpha}(f)$. In particular, $\mathbf{\Lambda}_{a, p}^{\alpha}$ and $\mathbf{\Lambda}_{a^{\prime}, p^{\prime}}^{-\alpha}$ are dual Banach spaces when $1<a<\infty$ and $1<p<\infty$.

Proof. It follows from Proposition 5.1 and 5.3 that the norm $\|f\|$ given above is equivalent to $\||f|\|$ where

$$
\||f|\|=\sup \left\{\left|\int f(x) T^{\alpha}(\lambda) v(x) d x\right|:\|v\|_{a, p} \leqq 1\right\} .
$$

Interchanging orders of integration, we get

$$
\||f|\|=\sup \left\{\left|\int T_{\alpha}(\lambda) f(x ; t) v(x ; t) d x d t\right|:\|v\|_{a, p} \leqq 1\right\} .
$$

Therefore $\|f\|\|=\| T_{\alpha}(\lambda) f \|_{a^{\prime}, p^{\prime}}$; we have taken for granted the fact that $\mathfrak{L}_{p}\left(\mathbf{L}_{a}\right)$ and $\mathcal{L}_{p^{\prime}}\left(\mathbf{L}_{a^{\prime}}\right)$ have dual norms. Using Proposition 5.2 and 5.3, we see that $\left\|T_{\alpha}(\lambda) f\right\|_{a^{\prime}, p^{\prime}}$ is equivalent to $\Lambda_{a^{\prime}, p^{\prime}}^{-\alpha}(f)$. Thus $\|f\|$ is equivalent to $\Lambda_{a^{\prime}, p^{\prime}}^{-\alpha}(f)$. When $1<a<\infty$ and $1<p<\infty$, the spaces $\mathfrak{L}_{p}\left(\mathbf{L}_{a}\right)$ and $\mathfrak{L}_{p^{\prime}}\left(\mathbf{L}_{a^{\prime}}\right)$ are dual to each other. When $a=1$ or $\infty$, or $p=1$ or $\infty$ this is no longer true, and slight complications arise, e.g. for $\alpha<0,1<a<\infty$, the dual space of $\mathbf{\Lambda}_{a, 1}^{\alpha}$ consists of the measurable functions $f$ such that $\Lambda_{a^{\prime}, \infty}^{-\alpha}(f)=\sup _{h}|h|^{\alpha}\left\|\Delta^{k}(h) f\right\|_{a^{\prime}}$ is finite.

The facts about interpolation of $\mathbf{\Lambda}_{a, p}^{\alpha}$-spaces are now easy to obtain.

Proposition 7.2. Suppose $0<\sigma<1$ and

$$
\gamma=\sigma \alpha+(1-\sigma) \beta, \quad 1 / c=\sigma / a+(1-\sigma) / b, \quad 1 / r=\sigma / p+(1-\sigma) / q .
$$


Then $\mathbf{\Lambda}_{c, r}^{\gamma}$ is the complex intermediate space $\left(\mathbf{\Lambda}_{a, p}^{\alpha}\right)^{\sigma}\left(\mathbf{\Lambda}_{b, q}^{\beta}\right)^{1-\sigma}$.

Proof. For a fixed $\lambda$, the transformations $T^{\alpha}(\lambda)$ of Proposition 5.1 are holomorphic in $\alpha$. Therefore, from the mappings

$$
T^{\alpha}(\lambda): \mathfrak{L}_{p}\left(\mathbf{L}_{a}\right) \rightarrow \mathbf{\Lambda}_{a, p}^{\alpha} ; \quad T^{\beta}(\lambda): \mathfrak{L}_{a}\left(\mathbf{L}_{b}\right) \rightarrow \mathbf{\Lambda}_{b, a}^{\beta}
$$

we have, according to the Riesz-Thorin convexity theorem

$$
T^{\alpha}(\lambda): \mathscr{L}_{r}\left(\mathbf{L}_{c}\right) \rightarrow\left(\mathbf{\Lambda}_{a, p}^{\alpha}\right)^{\sigma}\left(\mathbf{\Lambda}_{b, q}^{\beta}\right)^{1-\sigma}=\mathbf{X} .
$$

Proposition 5.3 says that $T^{\alpha}(\lambda) \mathscr{L}_{r}\left(\mathbf{L}_{c}\right)=\mathbf{\Lambda}_{c, r}^{\gamma}$, hence $\mathbf{\Lambda}_{c, r}^{\gamma} \subset \mathbf{X}$. This last inclusion implies that the intermediate-space norm is weaker than the $\mathbf{\Lambda}_{c, r^{-}}^{\gamma}$ norm. Put $\mathbf{Y}=\left(\boldsymbol{\Lambda}_{a^{\prime}, p^{\prime}}^{-\alpha}\right)^{\sigma}\left(\boldsymbol{\Lambda}_{b^{\prime}, \alpha^{\prime}}^{-\beta}\right)^{1-\sigma}$. The same argument gives $\boldsymbol{\Lambda}_{c^{\prime}, r^{\prime}}^{-\gamma} \subset \mathbf{Y}$, and so, by Proposition 7.1, the $\boldsymbol{\Lambda}_{c, r}^{\gamma}$-norm is stronger than the $\mathbf{Y}^{\prime}$-norm. Now, although we can only assert the inclusion $\mathrm{X} \subset \mathrm{Y}^{\prime}$ and not equality in general, the $\mathrm{Y}^{\prime}$-norm is equivalent to the $\mathrm{X}$-norm on $\mathrm{X}$. It follows that the $\mathrm{X}$-norm is equivalent to the $\boldsymbol{\Lambda}_{c, r}^{\gamma}$-norm, and this proves the assertion.

8. Sobolev's Lemma. The space $\mathbf{L}_{a}^{(\alpha)}$ is defined as the completion of $\hat{\theta}$ for the norm $\|f\|=\left\|R^{-\alpha} f\right\|_{a}$. For $1<a<\infty$ one can say that $f \varepsilon \mathbf{L}_{a}^{(\alpha)}$ means that the $\alpha$ th order derivatives of $f$ are in $\mathrm{L}_{a}$. The cases $a=1$ and $a=\infty$ present certain complications connected with Hilbert transforms. Also, $\mathbf{L}_{a}^{(0)}=\mathbf{L}_{a}$ for $1<a<\infty$, but $\mathbf{L}_{\infty}^{(0)}=\mathrm{C}_{0}$ and $\mathrm{L}_{1}^{(0)}$ consists of the functions in $\mathbf{L}_{1}$ whose integral is zero.

The following results describe the relationships between the $\mathbf{L}_{a}^{(\alpha)}$ spaces and the Lipschitz spaces. We shall always have $a, b, \alpha, \beta$ connected by

$$
1 / a-\alpha / n=1 / b-\beta / n, \quad 1 \leqq a \leqq b \leqq \infty .
$$

Theorem 4. If $a<b<\infty$ and $1 \leqq p \leqq b$ then

$$
\mathbf{\Lambda}_{a, p}^{\alpha} \subset \mathbf{L}_{b}^{(\beta)} \text {. }
$$

The inclusion persists for $a=b<\infty$ provided $p \leqq \min (b, 2)$. When $a \leqq b=\infty$ the statement is $\mathbf{\Lambda}_{a, 1}^{\alpha} \subset \mathbf{C}_{0}^{(\beta)}$.

Theorem $4^{\prime}$. If $1<a<b$ and $a \leqq q \leqq \infty$ then

$$
\mathbf{L}_{a}^{(\alpha)} \subset \mathbf{\Lambda}_{b, a}^{\beta} \text {. }
$$

The inclusion persists for $1<a=b$ provided $q \geqq \max (a, 2)$. When $1=a \leqq b$ the statement is $\mathrm{L}_{1}^{(\alpha)} \subset \mathbf{\Lambda}_{b, \infty}^{\beta}$.

Using Proposition 7.1 we see that the statements of Theorem 4 and $4^{\prime}$ correspond in dual pairs.

Corollary. If $1<a \leqq b<\infty$ then $\mathrm{L}_{a}^{(\alpha)} \subset \mathrm{L}_{b}^{(\beta)}$.

Proof. Given $a<b$ choose $c$ such that $a<c<b$ and $\gamma$ such that $1 / a-$ $\alpha / n=1 / c-\gamma / n=1 / b-\beta / n$. Theorem 4 gives $\Lambda_{c, c}^{\gamma} \subset \mathbf{L}_{b}^{(\beta)}$ while Theorem $4^{\prime}$ yields $\mathbf{L}_{a}^{(\alpha)} \subset \mathbf{\Lambda}_{c, c}^{\gamma}$. 
Theorem 4 lists all the valid inclusion relations of the form $\mathbf{\Lambda}_{a, p}^{\alpha} \subset \mathbf{L}_{b}^{(\beta)}$. Since the Riesz operators $R^{-\beta}$ give bijections it suffices to consider only the case $\beta=0$. When $\alpha>0$ a much more precise statement is available if one introduces Lorentz Spaces.

Theorem 5. If $1 / a-\alpha / n=1 / b>0$ and $\alpha>0$ then for all $p, 1 \leqq p \leqq \infty$,

$$
\mathbf{\Lambda}_{a, p}^{\alpha} \subset \mathbf{L}_{b, p} .
$$

Proof. According to Theorem 2 we have for $f \varepsilon \mathbf{\Lambda}_{a, p}^{\alpha}$,

$$
f(x)=\int_{0}^{\infty} t^{\alpha} g(x ; t) d t
$$

where $g(x ; t)=\kappa_{t} * u(x ; t)$ and $\|u(\cdot, t)\|_{a}=\omega(t)$ with $\omega \varepsilon \mathfrak{L}_{p}$. Taking averaged decreasing rearrangements we get

with majorizations

$$
f^{* *}(s) \leqq \int t^{\alpha} g^{* *}(s ; t) d t
$$

$$
\begin{aligned}
g^{* *}(s ; t) & \leqq\|g(\cdot, t)\|_{\infty}=\left\|\kappa_{t} * u(\cdot ; t)\right\|_{\infty} \leqq\left\|\kappa_{t}\right\|_{a} \\
\|u(\cdot ; t)\|_{a} & =t^{-n / a}\|\kappa\|_{a^{\prime}} \omega(t), \\
s^{1 / a} g^{* *}(s ; t) & \leqq\|g(\cdot ; t)\|_{a} \leqq\left\|\kappa_{t}\right\|_{1}\|u(\cdot ; t)\|_{a}=\|\kappa\|_{1} \omega(t) .
\end{aligned}
$$

It follows that

$$
s^{1 / b} f^{* *}(s) \leqq \int K\left(s t^{-n}\right) \omega(t) d t
$$

where

$$
K(s)=\|\kappa\|_{a^{\prime}} s^{1 / b} \quad \text { if } s<1,=\|\kappa\|_{1} s^{-\alpha / n} \quad \text { if } s>1 .
$$

Since $K \varepsilon \mathscr{L}_{1}$, convolution (on the multiplicative group $(0, \infty)$ ) with $K$ transforms $\mathscr{L}_{p}$ into $\mathscr{L}_{p}$, but to say $s^{1 / b} f^{* *} \varepsilon \mathscr{L}_{p}$ is to say $f \varepsilon \mathrm{L}_{b, p}$.

The assertions of Theorems 4 and $4^{\prime}$ which are not consequences of Theorem 5 are contained in the next three propositions. To get started we use

Lemma 8.1. The Fourier transform gives an isomorphism of $\mathbf{\Lambda}_{2, p}^{0}$ with $\mathbf{K}_{2, p}$ for all $p, 1 \leqq p<\infty$. In particular, $\mathbf{\Lambda}_{2,2}^{0}=\mathrm{L}_{2}$.

Proof. This follows from the case $\alpha=0$ of Proposition 3.1 and 3.1'. To see matters directly observe that

$$
\left\|\kappa_{t} * f\right\|_{2}=\left\|\hat{\kappa}^{t} \hat{f}\right\|_{2} .
$$

Proposition 8.1. If $1 \leqq a \leqq 2$ then $\mathbf{\Lambda}_{a, a}^{0} \subset \mathbf{L}_{a}$.

Proof. It is obvious from Theorem $2^{*}$ that $\mathbf{\Lambda}_{1,1}^{0} \subset \mathrm{L}_{1}$. Lemma 8.1 gives $\mathbf{\Lambda}_{\mathbf{2}, 2}^{0} \subset \mathbf{L}_{\mathbf{2}}$. The general statement follows by interpolation using Proposition 7.2 . 
Remark. When $1<a<2$ one may ask about an inclusion $\mathbf{\Lambda}_{a, p}^{0} \subset \mathbf{L}_{b, q}$. We can prove that $a=b \leqq q$ and $p<2$ are necessary conditions. The only open question is, therefore, whether there can be an inclusion $\mathbf{\Lambda}_{a, p}^{0} \subset \mathbf{L}_{a, a}$ with $a<p<2$.

Proposition 8.2. Inclusion relations $\mathbf{\Lambda}_{a, p}^{0} \subset \mathbf{C}_{0} \subset \mathbf{\Lambda}_{b, a}^{0}$ hold iff $a=b=$ $q=\infty$ and $p=1$.

Proof. It is immediate from Theorem $2^{*}$ that $\mathbf{\Lambda}_{\infty, 1}^{0} \subset \mathbf{C}_{0}$ and $\mathbf{\Lambda}_{1,1}^{0} \subset \mathbf{L}_{1}$. Dualizing the second of these inclusions we get $\mathbf{C}_{0} \subset \mathbf{\Lambda}_{\infty, \infty}^{0}$. It is clear from homogeneity considerations that the conditions $a=b=\infty$ are necessary. According to the remark after Proposition 1.2, $\boldsymbol{\Lambda}_{1, p}^{n}$ contains discontinuous functions if $p>1$; a fortiori, $\mathbf{\Lambda}_{\infty, p}^{0} \subset \mathbf{C}_{0}$, but also $\mathbf{\Lambda}_{1, p}^{0} \subset \mathbf{L}_{1}$ if $p>1$, for if we had $\mathbf{\Lambda}_{1, p}^{0} \subset \mathbf{L}_{1}$ we should have that functions in $\mathbf{\Lambda}_{1, p}^{n}$ have their $n^{\text {th }}$ derivatives in $\mathbf{L}_{1}$ and hence be continuous. Since $\mathbf{\Lambda}_{1, p}^{0} \subset \mathbf{L}_{1}$ for $p>1$, it follows by duality that $\mathbf{C}_{0} \subset \mathbf{\Lambda}_{\infty, a}^{0}$ for $q<\infty$.

Proposition 8.3. Suppose $1<a \leqq 2$. There is an inclusion $\mathbf{L}_{a, p} \subset \mathbf{\Lambda}_{b, a}^{0}$ iff $a=b, p \leqq a$, and $q \geqq 2$.

The affirmative assertion of Proposition 8.3 is

Lemma 8.2. For $1<a \leqq 2$, we have $\mathbf{L}_{a} \subset \mathbf{\Lambda}_{a, 2}^{0}$.

Proof. Given $f \varepsilon \mathbf{L}_{a}$, define the Marcinkiewicz Function of $f$ to be $\mu$ where

$$
\mu(x)=\left\{\int\left|\kappa_{t} * f(x)\right|^{2} d t\right\}^{1 / 2}
$$

The basic theorem on the Marcinkiewicz Function, cf. [4, Theorem 3.5], there exists a constant $M_{a}$ such that $\|\mu\|_{a} \leqq M_{a}\|f\|_{a}$ for all $a, 1<a<\infty$. Now for $a \leqq 2$, the $\mathbf{L}_{a}\left(\mathfrak{L}_{2}\right)$-norm of $\kappa_{t} * f(x)$ dominates the $\mathfrak{L}_{2}\left(\mathbf{L}_{a}\right)$-norm. This is to say that $\Lambda_{a, 2}^{0}(f) \leqq M_{a}\|f\|_{a}$.

The statement of Lemma 8.2 might appear to be a weak conclusion from its proof. Nevertheless we have

Lemma 8.3. If $p<2$ then $\mathbf{L}_{1} \cap \mathbf{C}_{0} \subset \Lambda_{a, p}^{0}$ for any a.

Proof. In the proof of Proposition 3.5 we found an $f \varepsilon \mathbf{L}_{1} \cap \mathbf{C}_{0}$ such that $\hat{f}$ was lacunary and $\hat{f} \notin \mathbf{L}_{p}$ for any $p<2$. If for this $f$ we had $f \varepsilon \mathbf{\Lambda}_{a, p}^{0}$ for some $a \leqq 2$ and $p<2$, then by Proposition 3.1 we should have $\hat{f} \varepsilon \mathbf{K}_{a^{\prime}, p}$, but since $\hat{f}$ is lacunary this would give $\hat{f} \varepsilon \mathbf{K}_{p, p}=\mathbf{L}_{p}$, a contradiction. The restriction $a \leqq 2$ is readily removed: if $a>2$ then $f \varepsilon \mathbf{\Lambda}_{a^{\prime}, 2}^{0}$ by Lemma 8.2, hence $f \varepsilon \mathbf{\Lambda}_{2, \alpha}^{0}$ where $q=4 p /(p+2)$ by Proposition 7.2, and $q<2$ if $p<2$.

Lemma 8.4. A necessary condition for an inclusion $\mathbf{\Lambda}_{a, p}^{0} \subset \mathbf{L}_{b, q}$ is $a=b \leqq q$.

Proof. The fact that $a=b$ follows from Lemma 1.2, namely $\Lambda_{a, p}^{0}\left(f^{t}\right)=$ $t^{-n / a} \Lambda_{a, p}^{0}(f)$, and $\left\|f^{t}\right\|_{b, a}=t^{-n / b}\|f\|_{b, a}$. Now suppose $\mathbf{\Lambda}_{a, 1}^{0} \subset \mathbf{L}_{a, a}$. We have to show that $q<a$ yields a contradiction. Consider first the case $a=2$. Ac- 
cording to Lemma 8.1, the Fourier transform maps $\mathbf{K}_{2,1}$ into $\mathbf{\Lambda}_{2,1}^{0}$. Hence the Fourier transform maps $\mathbf{K}_{2,1}$ into $\mathbf{L}_{2, a}$, but if $f$ has compact support disjoint from the origin and $f \varepsilon \mathbf{L}_{2}$ then $f \varepsilon \mathrm{K}_{2,1}$. We conclude from translation invariance that $\hat{f} \varepsilon \mathbf{L}_{2, q}$ for any $f \varepsilon \mathbf{L}_{2}$ with compact support. This implies $q \geqq 2$. The next case is $2<a<\infty$. Since $\mathbf{\Lambda}_{a^{\prime}, 1}^{0} \subset \mathbf{L}_{a^{\prime}, a^{\prime}}$ by Proposition 8.1, if also $\mathbf{\Lambda}_{a, 1}^{0} \subset \mathbf{L}_{a, a}$, then $\mathbf{\Lambda}_{2,1}^{0} \subset L_{2, p}$ where $2 / p=1 / a^{\prime}+1 / q$. Since we must have $p \leqq 2$ it follows that $q \geqq a$. The remaining case is $1<a<2(a=1$ and $a=\infty$ have been disposed of in Proposition 8.2). Dualizing Lemma 8.2 we have $\mathbf{\Lambda}_{a^{\prime}, 1}^{0} \subset \mathbf{\Lambda}_{a^{\prime}, 2}^{0} \subset$ $\mathbf{L}_{a^{\prime}, a^{\prime}}$. If $\mathbf{\Lambda}_{a, 1}^{0} \subset \mathbf{L}_{a, q}$ then the previous interpolation argument gives $\mathbf{\Lambda}_{2,1} \subset$ $\mathbf{L}_{2, p}$ where again $2 / p=1 / a^{\prime}+1 / q$.

Lemmas 8.2, 8.3, and the dual of 8.4 combine to give Proposition 8.3.

\section{Appendix 1.}

Proof of Lemma 1.1. Let $\Lambda$ and $\Lambda^{\prime}$ be $\Lambda_{X, p}^{\alpha}$ norms defined by using differences of orders $k$ and $k^{\prime}$ respectively. If $k^{\prime}>k$ then, since $X$ is a translation-invariant norm, $X\left\{\Delta^{k^{\prime}}(h) f\right\} \leqq 2^{k^{\prime}-k} X\left\{\Delta^{k}(h) f\right\}$. Hence, $\Lambda^{\prime} \leqq 2^{k^{\prime}-k} \Lambda$ whenever $k^{\prime}>k$.

To show the equivalence of the $\Lambda$ and $\Lambda^{\prime}$ norms in general, it remains only to prove that

$$
\Lambda^{\prime} \leqq c_{k}\left(1-2^{\alpha-k}\right)^{-1} \Lambda \text { when } k^{\prime}=k+1 \text { and } k>\alpha .
$$

Our demonstration is essentially combinatorial, and it has, perhaps, some independent interest.

Consider the identity

$$
(T+1)^{k}=\{2+(T-1)\}^{k}=2^{k}+(T-1) \sum_{j=0}^{k-1} a_{i} T^{j},
$$

where $a_{0}, \cdots a_{k-1}$ are certain numerical functions of $k$ which we shall not bother to calculate explicitly. We get

$$
\left(T^{2}-1\right)^{k}=2^{k}(T-1)^{k}+(T-1)^{k+1} \sum_{j=0}^{k-1} a_{j} T^{j}
$$

which we rewrite as

$$
2^{k}(T-1)^{k}=\left(T^{2}-1\right)^{k}-(T-1)^{k+1} \sum_{j=0}^{k-1} a_{i} T^{i} .
$$

Iterating this identity, we get for any natural number $m$,

$$
2^{m k}(T-1)^{k}=\left(T^{2^{m}}-1\right)^{k}-\sum_{i=0}^{m-1} 2^{(m-i) k}\left(T^{2^{i}}-1\right)^{k+1} \sum a_{i} T^{2^{i} j} .
$$

Now interpret $T$ as the translation operator $T(h)$. The identity becomes

$$
2^{m k} \Delta^{k}(h) f(x)=\Delta^{k}\left(2^{m} h\right) f(x)-\sum \sum 2^{(m-i) k} a_{j} \Delta^{k+1}\left(2^{i} h\right) f\left(x+2^{i} j h\right) .
$$

Writing $\omega(h)=X\left\{\Delta^{k}(h) f\right\}$ and $\omega^{\prime}(h)=X\left\{\Delta^{k+1}(h) f\right\}$ and using the translation invariance of $X$, we get

$$
2^{m k} \omega(h) \leqq \omega\left(2^{m} h\right)+c_{k} \sum_{i=0}^{m-1} 2^{(m-i) k} \omega^{\prime}\left(2^{i} h\right)
$$


where $c_{k}=\sum_{i=0}^{k-1}\left|a_{i}\right|$. This in turn yields

$$
2^{m k} \Lambda(f) \leqq 2^{m \alpha} \Lambda(f)+c_{k} \sum 2^{(m-i) k} \cdot 2^{i \alpha} \Lambda^{\prime}(f) .
$$

Dividing by $2^{m k}$ we get

$$
\Lambda(f) \leqq 2^{m(\alpha-k)} \Lambda(f)+c_{k} \sum_{i=0}^{m-1} 2^{i(\alpha-k)} \Lambda^{\prime}(f) .
$$

Now let $m$ tend to $\infty$. Since $k>\alpha$, the result is

$$
\Lambda(f) \leqq c_{k} \sum_{k=0}^{\infty} 2^{i(\alpha-k)} \Lambda^{\prime}(f) .
$$

Appendix 2. Bessel potentials. Lipschitz spaces are often, e.g. Taibleson [6], treated using the Bessel potentials $K^{\alpha}$ instead of the Riesz potentials $R^{\alpha}$. The Bessel potential is most conveniently recognized by its Fourier transform

$$
\hat{K}^{\alpha}(\xi)=\left(1+\pi|\xi|^{2}\right)^{-\alpha / 2},
$$

but for technical purposes another procedure is better. Put $G(x)=\exp \left(-\pi|x|^{2}\right)$. For $\alpha>0$ we have

$$
K^{\alpha}=2 \int e^{-t^{2}} G_{t} t^{\alpha} d t / \Gamma(\alpha / 2)
$$

a formula which is immediately deducible from the fact that $\hat{G}=G$ and $\hat{K}^{\alpha}=$ $2 \int e^{-t^{2}} G^{t} t^{\alpha} d t / \Gamma(\alpha / 2)$. The result which is essential for us is

Proposition. Suppose $0<\beta<\alpha$. Then $K^{\alpha} \varepsilon \Lambda_{1,1}^{\beta}$ and $\Lambda_{1, \infty}^{\alpha}\left(K^{\alpha}\right)<\infty$ although $K^{\alpha} \notin \mathbf{\Lambda}_{1, \infty}^{\alpha}$.

Proof. Since $K^{\alpha} * K^{\gamma}=K^{\alpha+\gamma}$, Lemma 1.3 reduces consideration to the case $0<\alpha<1$. Also, the fact that $G(x)=G\left(x_{1}\right) \cdots G\left(x_{n}\right)$ allows us to confine our attention to dimension $n=1$. For all $\beta>0, G \varepsilon \mathbf{\Lambda}_{1,1}^{\beta}$; so, writing $c_{\alpha}=$ $2 / \Gamma(\alpha / 2)$, we have

$$
\begin{aligned}
\Lambda_{1,1}^{\beta}\left(K^{\alpha}\right) & \leqq c_{\alpha} \int e^{-t^{2}} \Lambda_{1,1}^{\beta}\left(G_{t}\right) t^{\alpha} d t \\
& =c_{\alpha} \int e^{-t^{2}} \Lambda_{1,1}^{\beta}(G) t^{\alpha-\beta} d t<\infty, \text { when } \beta<\alpha .
\end{aligned}
$$

This proves that $K^{\alpha} \varepsilon \Lambda_{1,1}^{\beta}$ for $0<\beta<\alpha$. We cannot have $K^{\alpha} \varepsilon \mathbf{\Lambda}_{1, \infty}^{\alpha}$ since the Fourier transform of $R^{-\alpha} K^{\alpha}$ is $|\xi|^{\alpha}\left(1+\pi|\xi|^{2}\right)^{-\alpha / 2}$ while the Fourier transform maps $\mathbf{\Lambda}_{1, \infty}^{\mathbf{0}}$ into $\mathbf{C}_{0}$, functions tending to 0 at $\infty$. On the other hand, for $h>0$

$$
\begin{aligned}
\int\left|K^{\alpha}(x+h)-K^{\alpha}(x)\right| d x & \leqq c_{\alpha} \int e^{-t^{2}} t^{\alpha} \int\left|G_{t}(x+h)-G_{t}(x)\right| d x d t \\
& =2 c_{\alpha} \int e^{-t^{2}} t^{\alpha} \int_{0}^{h} G_{t}(x) d x d t
\end{aligned}
$$




$$
\begin{aligned}
& =2 c_{\alpha} \int e^{-t^{2}} t^{\alpha} \int_{0}^{t^{-1 h}} G(x) d x d t \\
& \leqq 2 c_{\alpha} \int t^{\alpha} \int_{0}^{t-1 h} G(x) d x d t \\
& =2 c_{\alpha} h^{\alpha} \int t^{\alpha} \int_{0}^{t-1} G(x) d x d t \\
& \leqq 2 c_{\alpha} h^{\alpha}\left\{\int_{0}^{1} t^{\alpha} d t+\int_{1}^{\infty} t^{\alpha-1} d t\right\} \\
& =2 c_{\alpha}\left\{\alpha^{-1}+(1-\alpha)^{-1}\right\} h^{\alpha} .
\end{aligned}
$$

This proves that $\Lambda_{1, \infty}^{\alpha}\left(K^{\alpha}\right)<\infty$.

A glance at the proof of Proposition 1.3 gives

Corollary. If $\alpha>0$ then $K^{\alpha} * \mathbf{\Lambda}_{b, a}^{\beta} \subset \mathbf{\Lambda}_{b, a}^{\alpha+\beta}$.

The spaces $\Lambda(\alpha ; a ; p)$ defined by Taibleson [6] are presented this way $(a, p \neq \infty)$ for $\alpha>0$,

$$
\Lambda(\alpha ; a ; p)=\mathbf{\Lambda}_{a, p}^{\alpha} \cap \mathbf{L}_{a}
$$

for all $\alpha, \beta$,

$$
K^{\alpha} * \Lambda(\beta ; b ; q)=\Lambda(\alpha+\beta ; b ; q) .
$$

It follows from Theorem 4 and the above corollary that

$$
\mathbf{\Lambda}_{a, p}^{0} \subset \Lambda(0 ; a ; p) \text { provided } p \leqq \min (a, 2) .
$$

This inclusion remains valid for $a=\infty, p=1$. The inclusions are reversed if $p \geqq \max (a, 2)$.

\section{Bibliography}

[1] Arne Beurling, Construction and analysis of some convolution algebras, Ann. l'Inst. Fourier, 14 (1964) 1-32.

[2] A. P. Calderón, Intermediate spaces and interpolation, Studia Math., 24 (1964) 113-190.

[3] C. HERz, The Ideal Theorem in certain Banach algebras of functions satisfying smoothness conditions, Gelbaum, editor, Functional Analysis, Thompson, Washington, D. C., 1967, 222-234.

[4] Lars Hörmander, Estimates for translation invariant operators in $L^{p}$ spaces, Acta Math., 104 (1960) 93-140.

[5] Richard A. Hunt, An extension of the Marcinkiewicz Interpolation Theorem to Lorentz spaces, Bull. Amer. Math. Soc., 70 (1964) 803-837.

[6] Mrtcheld H. Tarbleson, On the theory of Lipschitz spaces of distributions on Euclidean n-space, J. Math. Mech., 13 (1964) 407-408, 14 (1965) 821-839, and 15, (1966) 973-981.

Cornell University

Date communicated: ОстовER 10, 1967 